\title{
A Parametric Finite Element Method for Fourth Order Geometric Evolution Equations
}

\author{
John W. Barrett ${ }^{\dagger} \quad$ Harald Garcke Robert Nürnberg $^{\dagger}$
}

\begin{abstract}
We present a finite element approximation of motion by minus the Laplacian of curvature and related flows. The proposed scheme covers both the closed curve case, and the case of curves that are connected via triple junctions. On introducing a parametric finite element approximation, we prove stability bounds and compare our scheme with existing approaches. It turns out that the new scheme has very good properties with respect to area conservation and the equidistribution of mesh points. We state also an extension of our scheme to Willmore flow of curves and discuss possible further generalizations.
\end{abstract}

Key words. surface diffusion, Willmore flow, triple junctions, fourth order parabolic problem, parametric finite elements, Schur complement, tangential movement

AMS subject classifications. 65M60, 65M12, 35K55

\section{Introduction}

In this paper we introduce a parametric finite element approximation for motion by surface diffusion with the possible inclusion of triple junctions. For a closed hypersurface $\Gamma$ in $\mathbb{R}^{d}$, which evolves in time, motion by surface diffusion is given by the following evolution law for the normal velocity $\mathcal{V}$, see (1.2),

$$
\mathcal{V}=-\Delta_{s} \varkappa
$$

where $\Delta_{s}$ is the surface Laplacian and $\varkappa$ is the sum of the principal curvatures of $\Gamma$. For later use we remark that for a parameterization $\vec{x}(\rho, t) \in \mathbb{R}^{d}$ of $\Gamma,(1.1)$ can be written as a system of second order equations:

$$
\mathcal{V}:=\vec{x}_{t} \cdot \vec{\nu}=-\Delta_{s} \varkappa, \quad \varkappa \vec{\nu}=\Delta_{s} \vec{x}
$$

\footnotetext{
${ }^{\dagger}$ Department of Mathematics, Imperial College, London, SW7 2AZ, U.K.

${ }^{\ddagger}$ NWF I - Mathematik, Universität Regensburg, 93040 Regensburg, Germany
} 

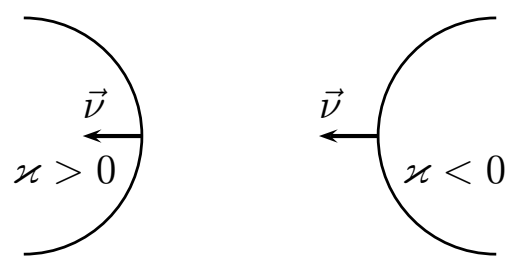

Figure 1: The sign convention for $\varkappa$.

where $\vec{\nu}$ is a unit normal to $\Gamma$. The second identity in (1.2) is well-known from surface geometry, and yields the convention that $\varkappa$ is positive if the curve is curved in the direction of the normal, see Figure 1 . Note that because the tangential component $\vec{x}_{t}-\left(\vec{x}_{t} \cdot \vec{\nu}\right) \vec{\nu}$ of the velocity $\vec{x}_{t}$ is not prescribed in (1.2), there exists a whole family of solutions $\vec{x}$, even though the evolution of $\Gamma$ is uniquely determined by (1.1).

The evolution law (1.1) was proposed by Mullins (1957) as an evolution law for a free surface enclosing a solid phase, which changes its shape due to the diffusion of atoms along the surface. Later a derivation, in the context of rational thermodynamics, was given by Davi and Gurtin (1990). Motion by surface diffusion governs the evolution of free surfaces in many applications such as e.g. thermal grooving, sintering, void evolution in microelectronic circuits and epitaxial growth; see e.g. Mullins (1957), Li, Zhao, and Gao (1999), Bower and Craft (1998), Fried and Gurtin (2003), Averbuch, Israeli, and Ravve (2003), and the references therein. Existence, uniqueness and stability results have been given by Elliott and Garcke (1997), Escher, Mayer, and Simonett (1998) and Escher, Garcke, and Ito (2003).

We remark that a surface that encloses a region in $\mathbb{R}^{d}$ and evolves according to (1.1) conserves volume. Choosing $\vec{\nu}$ to be the outward unit normal to the region and taking $a(t)$ as the total enclosed volume, this follows from

$$
\frac{d}{d t} a(t)=\int_{\Gamma} \mathcal{V} \mathrm{d} s=-\int_{\Gamma} \Delta_{s} \varkappa \mathrm{d} s=0
$$

where the last identity follows from the Gauss theorem on manifolds. Furthermore the total surface area, $|\Gamma(t)|$, decreases in time as can be seen from

$$
\frac{d}{d t}|\Gamma(t)|=-\int_{\Gamma} \varkappa \mathcal{V} \mathrm{d} s=\int_{\Gamma} \varkappa\left(\Delta_{s} \varkappa\right) \mathrm{d} s=-\int_{\Gamma}\left(\nabla_{s} \varkappa\right)^{2} \mathrm{~d} s \leq 0,
$$

where $\nabla_{s} f=\nabla f-(\vec{\nu} . \nabla f) \vec{\nu}$ is the tangential gradient on $\Gamma$, see e.g. Deckelnick, Dziuk, and Elliott (2005a, p. 150).

In this paper we will restrict our attention to the case $d=2$, i.e. curves in the plane. In many applications networks of curves with triple junctions appear. A model for surface diffusion of a network of curves has been introduced by Garcke and Novick-Cohen (2000), which we describe in the following for a network of three curves. Let $\Gamma_{1}, \Gamma_{2}, \Gamma_{3}$ be the given curves in $\mathbb{R}^{2}$ that intersect at two triple junction points $\Lambda_{1}$ and $\Lambda_{2}$; see Figure 2 . 
Let $\vec{\tau}_{i} \in \mathbb{R}^{2}$ be the unit tangent to $\Gamma_{i}$ pointing away from the triple junction point $\Lambda_{1}$ and towards point $\Lambda_{2}$. Then the normal velocity for each curve is given by

$$
\mathcal{V}_{i}=-\sigma_{i} \Delta_{s} \varkappa_{i}, \quad i=1 \rightarrow 3
$$

where $\varkappa_{i}$ is the curvature of $\Gamma_{i}$ and $\sigma_{i}$ is the surface energy density of $\Gamma_{i}$. The curvature is said to be positive if $\Gamma_{i}$ is curved in the direction of the normal $\vec{\nu}_{i} \in \mathbb{R}^{2}$, which is the unique unit vector that forms a positively orientated orthonormal system with $\vec{\tau}_{i}$. Then, in addition to (1.4), the following conditions have to hold at the triple junction points $\Lambda_{1}$ and $\Lambda_{2}$ :

the triple junction does not pull apart,

$$
\begin{aligned}
& \sigma_{1} \vec{\tau}_{1}+\sigma_{2} \vec{\tau}_{2}+\sigma_{3} \vec{\tau}_{3}=\overrightarrow{0}, \\
& \sigma_{1} \vec{\tau}_{1} \cdot \nabla_{s} \varkappa_{1}=\sigma_{2} \vec{\tau}_{2} \cdot \nabla_{s} \varkappa_{2}=\sigma_{3} \vec{\tau}_{3} \cdot \nabla_{s} \varkappa_{3}, \\
& \sigma_{1} \varkappa_{1}+\sigma_{2} \varkappa_{2}+\sigma_{3} \varkappa_{3}=0,
\end{aligned}
$$

where $\left.\nabla_{s}\right|_{\Gamma_{i}} \equiv \vec{\tau}_{i} \frac{\partial}{\partial s}$ with $s$ being the arclength chosen to be increasing in the direction of $\vec{\tau}_{i}$. The conditions $(1.5 \mathrm{a}-\mathrm{d})$ are an attachment condition, Young's law, a flux balance condition and a chemical potential continuity condition, respectively. Young's law (1.5b) is the force balance (leading to angle conditions) at the triple junction. If all surface energy densities, $\sigma_{i}$, are the same, then we recover the familiar $120^{\circ}$ degree condition. The flux balance condition (1.5c) follows from mass balance considerations at the triple junction. In order to be in thermodynamical equilibrium locally, it is necessary that the chemical potential differences are continuous which leads to $(1.5 \mathrm{~d})$; for more details on the above conditions see Garcke and Novick-Cohen (2000).

Taking the boundary conditions $(1.5 \mathrm{a}-\mathrm{d})$ into account, we easily derive that the total area of the enclosed phases are conserved; e.g. for the area $a_{3}(t)$ of the phase enclosed by $\Gamma_{1}$ and $\Gamma_{2}$, see Figure 2, we obtain that

$$
\frac{d}{d t} a_{3}(t)=\int_{\Gamma_{2}} \mathcal{V}_{2} \mathrm{~d} s-\int_{\Gamma_{1}} \mathcal{V}_{1} \mathrm{~d} s=-\sigma_{2} \int_{\Gamma_{2}} \Delta_{s} \varkappa_{2} \mathrm{~d} s+\sigma_{1} \int_{\Gamma_{1}} \Delta_{s} \varkappa_{1} \mathrm{~d} s=0,
$$

where the last identity follows from (1.5c). The total free energy of the system is given by $\sum_{i=1}^{3} \sigma_{i}\left|\Gamma_{i}(t)\right|$, where $\left|\Gamma_{i}(t)\right|$ is now the length of $\Gamma_{i}(t)$, and we obtain from

$$
\begin{aligned}
\frac{d}{d t} \sum_{i=1}^{3} \sigma_{i}\left|\Gamma_{i}(t)\right| & =-\sum_{i=1}^{3} \sigma_{i} \int_{\Gamma_{i}} \varkappa_{i} \mathcal{V}_{i} \mathrm{~d} s=\sum_{i=1}^{3} \sigma_{i}^{2} \int_{\Gamma_{i}} \varkappa_{i} \Delta_{s} \varkappa_{i} \mathrm{~d} s \\
& =-\sum_{i=1}^{3} \sigma_{i}^{2} \int_{\Gamma_{i}}\left|\nabla_{s} \varkappa_{i}\right|^{2} \mathrm{~d} s \leq 0
\end{aligned}
$$

that the total free energy cannot increase. The first identity above holds because of Young's law, (1.5b), and the last identity is true since the boundary conditions (1.5c) and (1.5d) imply that the boundary terms arising from the integration by parts disappear. 


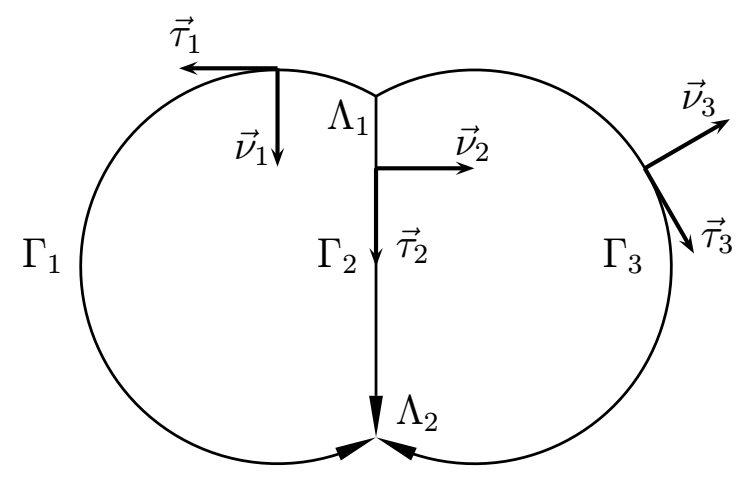

Figure 2: The setup of $\Gamma=\left(\Gamma_{1}, \Gamma_{2}, \Gamma_{3}\right)$.

For parameterizations $\vec{x}_{i} \in \mathbb{R}^{2}$ of $\Gamma_{i}, i=1 \rightarrow 3,(1.4)$ can be written as a system of second order equations:

$$
\left(\vec{x}_{i}\right)_{t} \cdot \vec{\nu}_{i}=-\sigma_{i} \Delta_{s} \varkappa_{i}, \quad \varkappa_{i} \vec{\nu}_{i}=\Delta_{s} \vec{x}_{i}
$$

A variational formulation of (1.6) will form the basis for our scheme that we present in Section 2.

Let us now shortly discuss existing numerical approaches to surface diffusion. Level set methods to compute surface diffusion and Willmore flow are studied in Chopp and Sethian (1999) and Droske and Rumpf (2004), respectively. Numerical approximations of parametric formulations of surface diffusion of closed curves (and surfaces) are the subject of the papers by Escher, Mayer, and Simonett (1998), Dziuk, Kuwert, and Schätzle (2002) and Bänsch, Morin, and Nochetto (2005). Our approach will use ideas of the latter two papers and of the seminal paper Dziuk (1991). We remark that all existing numerical approaches to the parametric formulation need to heuristically redistribute points tangentially in order to avoid coalescence of points. There are also numerical approaches for surface diffusion of axially symmetric surfaces, see Deckelnick, Dziuk, and Elliott (2003), and surface diffusion of graphs, see Bänsch, Morin, and Nochetto (2004) and Deckelnick, Dziuk, and Elliott (2005b). For an overview we refer to Deckelnick, Dziuk, and Elliott (2005a).

As for work on the approximation of curve networks, we refer to Bronsard and Wetton (1995), Thaddey (1999) and Neubauer (2002) for direct approximations of motion by mean curvature. A level set approach for mean curvature flow of curve networks has been considered in Merriman, Bence, and Osher (1994), Zhao, Merriman, Osher, and Wang (1998) and Smith, Solis, and Chopp (2002). A phase field approximation of the motion of surface diffusion of a closed curve was studied in Barrett, Nürnberg, and Styles (2004), and its extension to curve networks is given in Barrett, Garcke, and Nürnberg (2006a). In the present literature, to our knowledge, there is no work on the surface diffusion of a network of curves.

This paper is organised as follows. In Section 2, we formulate a finite element approximation of (1.6), (1.5a-d) and derive stability bounds. Here we first introduce our approximation for the simpler case of a closed curve, (1.2), and then generalize that 
scheme to cover (1.6) and (1.5a-d) in the case of a triple junction configuration as in Figure 2. In addition, we indicate how to generalize the approach to an arbitrary setup of curves and triple junctions. Moreover, we extend the scheme to approximate Willmore flow, and related elastic flows, for closed curves. In Section 3, we present some numerical computations for closed curves and compare our results with those from other algorithms in the literature. Furthermore, we include various numerical results on the triple junction setup of Figure 2 as well as on more general setups.

\section{Finite Element Approximation}

\subsection{Closed curves}

We introduce the following finite element approximation. Let $J:=\mathbb{R} / \mathbb{Z}=\cup_{j=1}^{N} J_{j}, N \geq 3$, be a decomposition of $J$ into intervals given by the nodes $q_{j}, J_{j}=\left[q_{j-1}, q_{j}\right]$. Here $J$ is the "periodic" interval [0,1], which we obtain by identifying points $q \in \mathbb{R}$ and $q+k$ for $k \in \mathbb{Z}$. Let $h_{j}=\left|J_{j}\right|$ and $h=\max _{j=1 \rightarrow N} h_{j}$ be the maximal length of a grid element. Then the necessary finite element spaces are defined as follows:

$$
\underline{V}_{0}^{h}:=\left\{\vec{\chi} \in C\left(J, \mathbb{R}^{2}\right):\left.\vec{\chi}\right|_{J_{j}} \text { is linear } \forall j=1 \rightarrow N\right\}=:\left[W_{0}^{h}\right]^{2} \subset H^{1}\left(J, \mathbb{R}^{2}\right),
$$

where $W_{0}^{h} \subset H^{1}(J, \mathbb{R})$ is the space of scalar continuous (periodic) piecewise linear functions, with $\left\{\phi_{l}\right\}_{l=1}^{N}$ denoting the standard basis of $W_{0}^{h}$. Throughout this paper, we make use of the periodicity of $J$, i.e. $q_{N} \equiv q_{0}, q_{N+1} \equiv q_{1}$ and so on.

In addition, let $0=t_{0}<t_{1}<\ldots<t_{M-1}<t_{M}=T$ be a partitioning of $[0, T]$ into possibly variable time steps $\tau_{m}:=t_{m+1}-t_{m}, m=0 \rightarrow M-1$. We set $\tau:=$ $\max _{m=0 \rightarrow M-1} \tau_{m}$. Let $\vec{X}^{m} \in \underline{V}_{0}^{h}$ be an approximation to $\vec{x}\left(\cdot, t_{m}\right)$, and similarly $\kappa^{m} \in W_{0}^{h}$ for $\varkappa\left(\cdot, t_{m}\right)$.

For scalar and vector functions $f, g \in L^{2}\left(J, \mathbb{R}^{(2)}\right)$ we introduce the $L^{2}$ inner product $\langle\cdot, \cdot\rangle_{m}$ over the current polygonal curve $\Gamma^{m}$, which is described by the vector function $\vec{X}^{m} \in \underline{V}_{0}^{h}$, as follows

$$
\langle f, g\rangle_{m}:=\int_{\Gamma^{m}} f \cdot g \mathrm{~d} s=\int_{J} f \cdot g\left|\vec{X}_{\rho}^{m}\right| \mathrm{d} \rho
$$

where in the first integral, and throughout this paper, for notational simplicity there is a slight abuse of notation. In addition, in the above and throughout this paper, $\rho \in$ $[0,1]$ is the parameterization variable and.$\left.^{*}\right)$ denotes an expression with or without the superscript $*$, and similarly for subscripts. In addition, if $f, g$ are piecewise continuous, with possible jumps at the nodes $\left\{q_{j}\right\}_{j=1}^{N}$, we define the mass lumped inner product $\langle\cdot, \cdot\rangle_{m}^{h}$ as

$$
\langle f, g\rangle_{m}^{h}:=\frac{1}{2} \sum_{j=1}^{N}\left|\vec{X}^{m}\left(q_{j}\right)-\vec{X}^{m}\left(q_{j-1}\right)\right|\left[(f . g)\left(q_{j}^{-}\right)+(f . g)\left(q_{j-1}^{+}\right)\right]
$$


where we define $f\left(q_{j}^{ \pm}\right):=\lim _{\varepsilon \searrow 0} f\left(q_{j} \pm \varepsilon\right)$. Furthermore, we note that

$$
\nabla_{s} f \cdot \nabla_{s} g=\frac{f_{\rho} \cdot g_{\rho}}{\left|\vec{X}_{\rho}^{m}\right|^{2}} \quad \text { and } \quad \vec{\nu}^{m}=-\frac{\left(\vec{X}_{\rho}^{m}\right)^{\perp}}{\left|\vec{X}_{\rho}^{m}\right|}
$$

where ${ }^{\perp}$ acting on $\mathbb{R}^{2}$ denotes clockwise rotation by $\frac{\pi}{2}$. We propose the following approximation to (1.2): Find $\left\{\vec{X}^{m+1}, \kappa^{m+1}\right\} \in \underline{V}_{0}^{h} \times W_{0}^{h}$ such that

$$
\begin{aligned}
& \left\langle\frac{\vec{X}^{m+1}-\vec{X}^{m}}{\tau_{m}}, \chi \vec{\nu}^{m}\right\rangle_{m}^{h}-\left\langle\nabla_{s} \kappa^{m+1}, \nabla_{s} \chi\right\rangle_{m}=0 \quad \forall \chi \in W_{0}^{h}, \\
& \left\langle\kappa^{m+1} \vec{\nu}^{m}, \vec{\eta}\right\rangle_{m}^{h}+\left\langle\nabla_{s} \vec{X}^{m+1}, \nabla_{s} \vec{\eta}\right\rangle_{m}=0 \quad \forall \vec{\eta} \in \underline{V}_{0}^{h}
\end{aligned}
$$

where, as noted above, the inner products $\langle\cdot, \cdot\rangle_{m}^{(h)}$ as well as $\nabla_{s}$ depend on $m$.

REMARK. 2.1. In Section 3, we will report on computations for the scheme (2.2a,b) and compare the results with two other schemes in the literature. The first is from Dziuk, Kuwert, and Schätzle (2002) and can be formulated as: Find $\left\{\vec{X}^{m+1}, \vec{\kappa}^{m+1}\right\} \in\left[\underline{V}_{0}^{h}\right]^{2}$ such that for all $\vec{\chi}, \vec{\eta} \in \underline{V}_{0}^{h}$

$$
\begin{array}{ll}
\left\langle\frac{\vec{X}^{m+1}-\vec{X}^{m}}{\tau_{m}}, \vec{\chi}\right\rangle_{m}^{h}-\left\langle\nabla_{s} \vec{\kappa}^{m+1}, \nabla_{s} \vec{\chi}\right\rangle_{m}-\frac{3}{2}\left\langle\left|\vec{\kappa}^{m}\right|^{2} \nabla_{s} \vec{X}^{m+1}, \nabla_{s} \vec{\chi}\right\rangle_{m} \\
\left\langle\vec{\kappa}^{m+1}, \vec{\eta}\right\rangle_{m}^{h}+\left\langle\nabla_{s} \vec{X}^{m+1}, \nabla_{s} \vec{\eta}\right\rangle_{m}=0 ; & -\frac{1}{2}\left\langle\left|\vec{\kappa}^{m}\right|^{2} \vec{\kappa}^{m+1}, \vec{\chi}\right\rangle_{m}^{h}=0
\end{array}
$$

where $\vec{\kappa}^{0} \in \underline{V}_{0}^{h}$ is such that

$$
\left\langle\vec{\kappa}^{0}, \vec{\eta}\right\rangle_{0}^{h}+\left\langle\nabla_{s} \vec{X}^{0}, \nabla_{s} \vec{\eta}\right\rangle_{0}=0 \quad \forall \vec{\eta} \in \underline{V}_{0}^{h} .
$$

The system $(2.3 \mathrm{a}, \mathrm{b})$ is a discretization of the variational formulation of

$$
\vec{x}_{t}=-\left(\Delta_{s} \varkappa\right) \vec{\nu} \equiv-\Delta_{s} \vec{\varkappa}-\frac{3}{2} \nabla_{s} \cdot\left(|\vec{\varkappa}|^{2} \nabla_{s} \vec{x}\right)+\frac{1}{2}|\vec{\varkappa}|^{2} \vec{\varkappa}, \quad \vec{\varkappa}:=\varkappa \vec{\nu}=\Delta_{s} \vec{x}
$$

as opposed to (1.2). The second scheme is from Bänsch, Morin, and Nochetto (2005) and can be stated as: Let $\vec{X}^{m+1}:=\vec{X}^{m}+\tau_{m} \vec{V}^{m+1}$, where $\vec{V}^{m+1} \in \underline{V}_{0}^{h}$ is part of the solution of: Find $\left\{\vec{V}^{m+1}, \kappa^{m+1}, \vec{\kappa}^{m+1}, V^{m+1}\right\} \in \underline{V}_{0}^{h} \times W_{0}^{h} \times \underline{V}_{0}^{h} \times W_{0}^{h}$ such that

$$
\begin{aligned}
\left\langle\vec{\kappa}^{m+1}, \vec{\eta}\right\rangle_{m}+\tau_{m}\left\langle\nabla_{s} \vec{V}^{m+1}, \nabla_{s} \vec{\eta}\right\rangle_{m} & =-\left\langle\nabla_{s} \vec{X}^{m}, \nabla_{s} \vec{\eta}\right\rangle_{m} & & \forall \vec{\eta} \in \underline{V}_{0}^{h}, \\
\left\langle\kappa^{m+1}, \chi\right\rangle_{m}-\left\langle\vec{\kappa}^{m+1}, \chi \vec{\nu}^{m}\right\rangle_{m} & =0 & & \forall \chi \in W_{0}^{h}, \\
\left\langle V^{m+1}, \chi\right\rangle_{m}-\left\langle\nabla_{s} \kappa^{m+1}, \nabla_{s} \chi\right\rangle_{m} & =0 & & \forall \chi \in W_{0}^{h}, \\
\left\langle\vec{V}^{m+1}, \vec{\eta}\right\rangle_{m}-\left\langle V^{m+1} \vec{\nu}^{m}, \vec{\eta}\right\rangle_{m} & =0 & & \forall \vec{\eta} \in \underline{V}_{0}^{h} .
\end{aligned}
$$

The system $(2.4 \mathrm{a}-\mathrm{d})$ is a discretization of the variational formulation of

$$
\vec{\varkappa}=\Delta_{s} \vec{x}, \quad \varkappa=\vec{\varkappa} \cdot \vec{\nu}, \quad v=-\Delta_{s} \varkappa, \quad \vec{x}_{t}=\vec{v}=v \vec{\nu}
$$




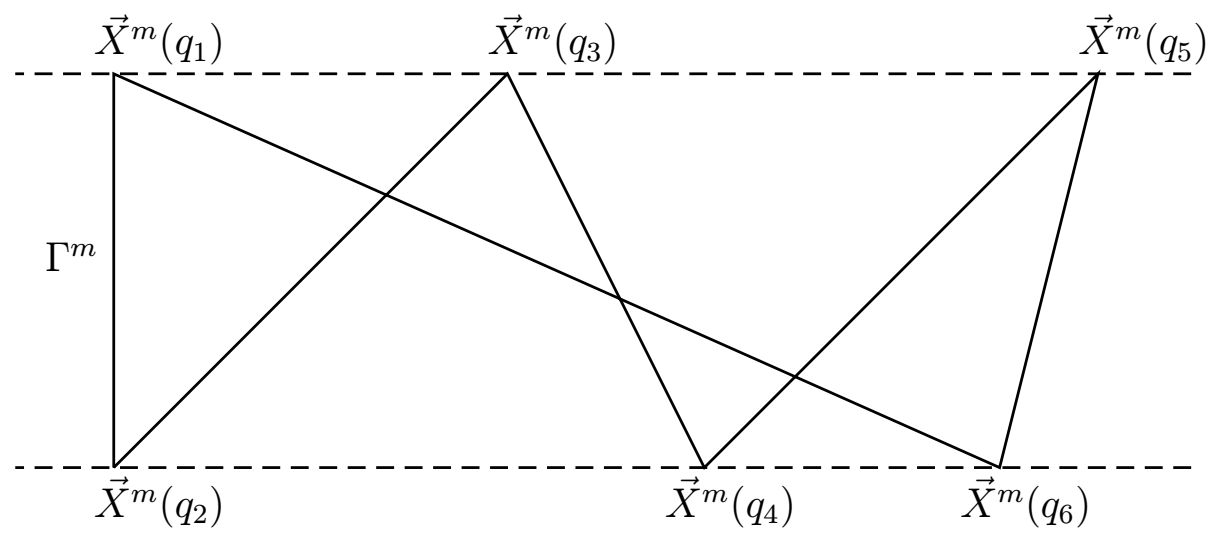

Figure 3: $\left(\mathcal{A}_{0}\right)$ is violated in this case.

as opposed to (1.2). We note that both schemes (2.3a,b) and (2.4a-d) only change the approximation of $\vec{x}$ in the normal direction, whereas the scheme $(2.2 \mathrm{a}, \mathrm{b})$ proposed in this paper also induces tangential changes. This is a crucial difference. Finally, it is shown in Bänsch, Morin, and Nochetto (2005) that (2.4a-d) satisfies a discrete analogue of (1.3). We will prove the analogous result for $(2.2 \mathrm{a}, \mathrm{b})$, see Theorem 2.3, and the ensuing comment, below.

Before we can proceed to prove existence and uniqueness to $(2.2 \mathrm{a}, \mathrm{b})$, we have to make the following very mild assumption.

$\left(\mathcal{A}_{0}\right)$ Let $\left|\vec{X}_{\rho}^{m}\right|>0$ for almost all $\rho \in J$. For $j=1 \rightarrow N$, let $\vec{\nu}_{j-\frac{1}{2}}^{m}:=-\left.\frac{\left(\vec{X}_{\rho}^{m}\right)^{\perp}}{\left|\vec{X}_{\rho}^{m}\right|}\right|_{J_{j}}$, and set

$$
\begin{aligned}
\vec{\omega}_{j}^{m} & :=\frac{\left|\vec{X}^{m}\left(q_{j}\right)-\vec{X}^{m}\left(q_{j-1}\right)\right| \vec{\nu}_{j-\frac{1}{2}}^{m}+\left|\vec{X}^{m}\left(q_{j+1}\right)-\vec{X}^{m}\left(q_{j}\right)\right| \vec{\nu}_{j+\frac{1}{2}}^{m}}{\left|\vec{X}^{m}\left(q_{j}\right)-\vec{X}^{m}\left(q_{j-1}\right)\right|+\left|\vec{X}^{m}\left(q_{j+1}\right)-\vec{X}^{m}\left(q_{j}\right)\right|} \\
& =\frac{-\left[\vec{X}^{m}\left(q_{j+1}\right)-\vec{X}^{m}\left(q_{j-1}\right)\right]^{\perp}}{\left|\vec{X}^{m}\left(q_{j}\right)-\vec{X}^{m}\left(q_{j-1}\right)\right|+\left|\vec{X}^{m}\left(q_{j+1}\right)-\vec{X}^{m}\left(q_{j}\right)\right|}
\end{aligned}
$$

Then we further assume that $\operatorname{dim} \operatorname{span}\left\{\vec{\omega}_{j}^{m}\right\}_{j=1}^{N}=d=2$.

REMARK. 2.2. a) We note that one can interpret $\vec{\omega}_{j}^{m}$ as a weighted normal defined at the node $\vec{X}^{m}\left(q_{j}\right)$ of the curve $\Gamma^{m}$, where in general $\left|\vec{\omega}_{j}^{m}\right|<1$. Noting that $\vec{\omega}_{j}^{m}$ points in the direction $\left[\vec{X}^{m}\left(q_{j+1}\right)-\vec{X}^{m}\left(q_{j-1}\right)\right]^{\perp}$, we obtain that the the assumption $\left(\mathcal{A}_{0}\right)$ is equivalent to excluding the following situation: All points $\left\{\vec{X}^{m}\left(q_{j}\right): j\right.$ is even $\}$ lie on one straight line and simultaneously all points $\left\{\vec{X}^{m}\left(q_{j}\right): j\right.$ is odd $\}$ lie on another parallel line.

b) Since $\vec{X}^{m}\left(q_{N}\right)=\vec{X}^{m}\left(q_{0}\right)$, we obtain in the case that $N$ is odd that $\left(\mathcal{A}_{0}\right)$ immediately holds provided all points do not lie on one straight line. If $N$ is even, then $\left(\mathcal{A}_{0}\right)$ is only violated on very rare occasions, see e.g. Figure 3. For example, for closed curves $\Gamma^{m}$ without self intersections the assumption $\left(\mathcal{A}_{0}\right)$ always holds. 
THEOREM. 2.1. Let the assumption $\left(\mathcal{A}_{0}\right)$ hold. Then there exists a unique solution $\left\{\vec{X}^{m+1}, \kappa^{m+1}\right\} \in \underline{V}_{0}^{h} \times W_{0}^{h}$ to the system $(2.2 \mathrm{a}, \mathrm{b})$.

Proof. As $(2.2 \mathrm{a}, \mathrm{b})$ is linear, existence follows from uniqueness. To investigate the latter, we consider the system: Find $\{\vec{X}, \kappa\} \in \underline{V}_{0}^{h} \times W_{0}^{h}$ such that

$$
\begin{aligned}
\left\langle\vec{X}, \chi \vec{\nu}^{m}\right\rangle_{m}^{h}-\tau_{m}\left\langle\nabla_{s} \kappa, \nabla_{s} \chi\right\rangle_{m}=0 & \forall \chi \in W_{0}^{h}, \\
\left\langle\kappa \vec{\nu}^{m}, \vec{\eta}\right\rangle_{m}^{h}+\left\langle\nabla_{s} \vec{X}, \nabla_{s} \vec{\eta}\right\rangle_{m}=0 & \forall \vec{\eta} \in \underline{V}_{0}^{h} .
\end{aligned}
$$

Choosing $\chi=\kappa \in W_{0}^{h}$ in (2.6a) and $\vec{\eta}=\vec{X} \in \underline{V}_{0}^{h}$ in (2.6b) yields that

$$
\left\langle\nabla_{s} \vec{X}, \nabla_{s} \vec{X}\right\rangle_{m}+\tau_{m}\left\langle\nabla_{s} \kappa, \nabla_{s} \kappa\right\rangle_{m}=0
$$

It follows from (2.7) that $\kappa \equiv \kappa^{c} \in \mathbb{R}$ and $\vec{X} \equiv \vec{X}^{c} \in \mathbb{R}^{2}$; and hence that

$$
\left\langle\vec{X}^{c}, \chi \vec{\nu}^{m}\right\rangle_{m}^{h}=0 \quad \forall \chi \in W_{0}^{h}, \quad \kappa^{c}\left\langle\vec{\nu}^{m}, \vec{\eta}\right\rangle_{m}^{h}=0 \quad \forall \vec{\eta} \in \underline{V}_{0}^{h} .
$$

Choosing $\vec{\eta}=\vec{z} \phi_{j} \in \underline{V}_{0}^{h}$ in (2.8), and noting (2.1) and (2.5), yields, on assuming $\kappa^{c} \neq 0$, that for all $j=1 \rightarrow N$

$$
\begin{aligned}
& \frac{1}{2}\left[\left|\vec{X}^{m}\left(q_{j}\right)-\vec{X}^{m}\left(q_{j-1}\right)\right| \vec{\nu}_{j-\frac{1}{2}}^{m}+\left|\vec{X}^{m}\left(q_{j+1}\right)-\vec{X}^{m}\left(q_{j}\right)\right| \vec{\nu}_{j+\frac{1}{2}}^{m}\right] \cdot \vec{z}=0 \quad \forall \vec{z} \in \mathbb{R}^{2} \\
\Longleftrightarrow & \vec{\omega}_{j}^{m} \cdot \vec{z}=0 \quad \forall \vec{z} \in \mathbb{R}^{2} \quad \Longleftrightarrow \quad \vec{\omega}_{j}^{m}=\overrightarrow{0} .
\end{aligned}
$$

However, this contradicts assumption $\left(\mathcal{A}_{0}\right)$ and hence $\kappa^{c}=0$. Similarly, testing (2.8) with $\chi=\phi_{j}$ yields that $\vec{X}^{c} \cdot \vec{\omega}_{j}^{m}=0$ for all $j=1 \rightarrow N$. It follows from assumption $\left(\mathcal{A}_{0}\right)$ that $\vec{X}^{c}=\overrightarrow{0}$. Hence we have shown that $(2.2 \mathrm{a}, \mathrm{b})$ has a unique solution $\left\{\vec{X}^{m+1}, \kappa^{m+1}\right\} \in$ $\underline{V}_{0}^{h} \times W_{0}^{h}$.

Here and throughout, for a given $n \in \mathbb{N}$, let $\overrightarrow{I d}_{n} \in\left(\mathbb{R}^{2 \times 2}\right)^{n \times n}$ be the identity matrix, and similarly for $I d_{n} \in \mathbb{R}^{n \times n}$. We introduce also the matrices $\vec{N}_{0} \in\left(\mathbb{R}^{2}\right)^{N \times N}, A_{0} \in \mathbb{R}^{N \times N}$ and $\overrightarrow{A_{0}} \in\left(\mathbb{R}^{2 \times 2}\right)^{N \times N}$ with entries

$$
\left[\vec{N}_{0}\right]_{k l}:=\int_{\Gamma^{m}} \pi^{h}\left[\phi_{k} \phi_{l}\right] \vec{\nu}^{m} \mathrm{~d} s, \quad\left[A_{0}\right]_{k l}:=\left\langle\nabla_{s} \phi_{k}, \nabla_{s} \phi_{l}\right\rangle_{m}, \quad\left[\vec{A}_{0}\right]_{k l}:=\left[A_{0}\right]_{k l} \overrightarrow{I d}_{1},
$$

where $\pi^{h}: C(J, \mathbb{R}) \rightarrow W_{0}^{h}$ is the standard interpolation operator at the nodes $\left\{q_{j}\right\}_{j=1}^{N}$. We can then formulate $(2.2 \mathrm{a}, \mathrm{b})$ as: Find $\left\{\delta \vec{X}^{m+1}, \kappa^{m+1}\right\} \in\left(\mathbb{R}^{2}\right)^{N} \times \mathbb{R}^{N}$ such that

$$
\left(\begin{array}{cc}
\tau_{m} A_{0} & -\vec{N}_{0}^{T} \\
\vec{N}_{0} & \vec{A}_{0}
\end{array}\right)\left(\begin{array}{c}
\kappa^{m+1} \\
\delta \vec{X}^{m+1}
\end{array}\right)=\left(\begin{array}{c}
0 \\
-\vec{A}_{0} \vec{X}^{m}
\end{array}\right)
$$

where, with the obvious abuse of notation, $\delta \vec{X}^{m+1}=\left(\delta \vec{X}_{1}^{m+1}, \ldots, \delta \vec{X}_{N}^{m+1}\right)^{T}$ and $\kappa^{m+1}=$ $\left(\kappa_{1}^{m+1}, \ldots, \kappa_{N}^{m+1}\right)^{T}$ are the vectors of coefficients with respect to the standard basis of $\vec{X}^{m+1}-\vec{X}^{m}$ and $\kappa^{m+1}$, respectively. The discrete system arising from $(2.4 \mathrm{a}-\mathrm{d})$ is solved in Bänsch, Morin, and Nochetto (2005) using a Schur complement approach. We adopt 
a similar procedure here for (2.11). Let $S_{0}$ be the inverse of $A_{0}$ restricted on the set $\left(\operatorname{ker} A_{0}\right)^{\perp} \equiv(\operatorname{span}\{1\})^{\perp}$, where $1:=(1, \ldots, 1)^{T} \in \mathbb{R}^{N}$ and $\cdot^{\perp}$ acting on a space denotes its orthogonal complement. Then noting that the first equation in (2.11) implies that $1^{T} \vec{N}_{0}^{T} \delta \vec{X}^{m+1}=0$, one can transform $(2.11)$ to

$$
\begin{aligned}
\kappa^{m+1} & =\frac{1}{\tau_{m}} S_{0} \vec{N}_{0}^{T} \delta \vec{X}^{m+1}+\mu 1, \\
\left(\vec{A}_{0}+\frac{1}{\tau_{m}} \vec{N}_{0} S_{0} \vec{N}_{0}^{T}\right) \delta \vec{X}^{m+1} & =-\vec{A}_{0} \vec{X}^{m}-\mu \vec{N}_{0} 1, \quad\left(\delta \vec{X}^{m+1}\right)^{T} \vec{N}_{0} 1=0 ;
\end{aligned}
$$

where $\mu=\frac{1^{T} \kappa^{m+1}}{1^{T} 1} \in \mathbb{R}$ is unknown. We introduce also the orthogonal projection $\vec{\Pi}_{0}$ onto $\mathcal{R}_{0}^{\perp}:=\left\{\vec{X} \in\left(\mathbb{R}^{2}\right)^{N}: \vec{X}^{T} \vec{N}_{0} 1=0\right\}$ by $\vec{\Pi}_{0}:=\overrightarrow{I d}_{N}-\frac{\vec{w} \vec{w}^{T}}{\vec{w}^{T} \vec{w}}$, where $\vec{w}:=\vec{N}_{0} 1$. Then $(2.12 \mathrm{~b})$, on noting that $\vec{\Pi}_{0} \delta \vec{X}^{m+1}=\delta \vec{X}^{m+1}$, is replaced by

$$
\vec{\Pi}_{0}\left(\vec{A}_{0}+\frac{1}{\tau_{m}} \vec{N}_{0} S_{0} \vec{N}_{0}^{T}\right) \vec{\Pi}_{0} \delta \vec{X}^{m+1}=-\vec{\Pi}_{0} \vec{A}_{0} \vec{X}^{m}
$$

As $(2.2 \mathrm{a}, \mathrm{b})$ has a unique solution, it is easily established that there exists a unique solution to (2.13). Moreover, the system (2.13) is symmetric and positive definite on $\mathcal{R}_{0}^{\perp}$. For details we refer to the triple junction case which is handled in Theorem 2.4 below.

In addition, a stability result for $(2.2 \mathrm{a}, \mathrm{b})$ is easily established; see Theorem 2.3 , and the ensuing comment, below.

REMARK. 2.3. It is worthwhile to consider a continuous in time semidiscrete version of our scheme. Here we replace $(2.2 \mathrm{a}, \mathrm{b})$ by

$$
\begin{aligned}
& \left\langle\vec{X}_{t}, \chi \vec{\nu}^{h}\right\rangle^{h}-\left\langle\nabla_{s} \kappa, \nabla_{s} \chi\right\rangle=0 \quad \forall \chi \in W_{0}^{h}, \\
& \left\langle\kappa \vec{\nu}^{h}, \vec{\eta}\right\rangle^{h}+\left\langle\nabla_{s} \vec{X}, \nabla_{s} \vec{\eta}\right\rangle=0 \quad \forall \vec{\eta} \in \underline{V}_{0}^{h} ;
\end{aligned}
$$

where we always integrate over the current curve $\Gamma^{h}$, described by $\vec{X}$, and so $\vec{\nu}^{h}=-\frac{\left(\vec{X}_{\rho}\right)^{\perp}}{\left|\vec{X}_{\rho}\right|}$ and $\langle\cdot, \cdot\rangle^{(h)}$ is the same as $\langle\cdot, \cdot\rangle_{m}^{(h)}$ with $\Gamma^{m}$ and $\vec{X}^{m}$ replaced by $\Gamma^{h}$ and $\vec{X}$, respectively. It is now straightforward to show that $(2.14 \mathrm{a}, \mathrm{b})$ conserves the enclosed area, $a^{h}(t)$, exactly; since on choosing $\chi=1$ in (2.14a) and taking into account (2.1) yields that

$$
0=\left\langle\vec{X}_{t}, \vec{\nu}^{h}\right\rangle^{h}=\int_{\Gamma^{h}} \vec{X}_{t} \cdot \vec{\nu}^{h} \mathrm{~d} s=\frac{d}{d t} a^{h}(t) .
$$

To our knowledge, no other direct approximation of (1.2) in the literature satisfies this property. While it is not possible to prove an analogues of (2.15) for $(2.2 \mathrm{a}, \mathrm{b})$, in practice we observe that the enclosed area is approximately preserved, and that the area loss tends to zero as $\tau \rightarrow 0$.

REMARK. 2.4. Furthermore, the scheme $(2.14 \mathrm{a}, \mathrm{b})$ will always equidistribute the vertices along $\Gamma^{h}$ for $t>0$, provided that they are not locally parallel; since on choosing $\vec{\eta}=$ $\left(\vec{\omega}_{j}^{h}\right)^{\perp} \phi_{j} \in \underline{V}_{0}^{h}$ in $(2.14 \mathrm{~b})$, where $\vec{\omega}_{j}^{h}$ is the $\Gamma^{h}$ analogue of $\vec{\omega}_{j}^{m}$, yields, on recalling $(2.1)$ and (2.5), that for $j=1 \rightarrow N$

$$
\begin{aligned}
& {\left[\frac{\vec{X}_{j+1}-\vec{X}_{j}}{\left|\vec{X}_{j+1}-\vec{X}_{j}\right|}-\frac{\vec{X}_{j}-\vec{X}_{j-1}}{\left|\vec{X}_{j}-\vec{X}_{j-1}\right|}\right] \cdot\left(\vec{X}_{j+1}-\vec{X}_{j-1}\right)=0 \Rightarrow} \\
& {\left[\left|\vec{X}_{j+1}-\vec{X}_{j}\right|-\left|\vec{X}_{j}-\vec{X}_{j-1}\right|\right]\left[\left|\vec{X}_{j+1}-\vec{X}_{j}\right|\left|\vec{X}_{j}-\vec{X}_{j-1}\right|-\left(\vec{X}_{j+1}-\vec{X}_{j}\right) \cdot\left(\vec{X}_{j}-\vec{X}_{j-1}\right)\right]=0} \\
& \quad \Rightarrow \quad \text { either }\left|\vec{X}_{j+1}-\vec{X}_{j}\right|=\left|\vec{X}_{j}-\vec{X}_{j-1}\right| \text { or } \quad\left(\vec{X}_{j+1}-\vec{X}_{j}\right) \|\left(\vec{X}_{j}-\vec{X}_{j-1}\right), \quad(2.16)
\end{aligned}
$$


where, here and throughout, $\vec{X}_{j}^{(m)} \equiv \vec{X}^{(m)}\left(q_{j}\right)$.

Of course, the analysis in (2.16) immediately carries over to a fully discrete scheme that is fully implicit; that is, $\vec{\nu}^{m}$ and $\langle\cdot, \cdot\rangle_{m}^{(h)}$ in $(2.2 \mathrm{a}, \mathrm{b})$ are replaced by $\vec{\nu}^{m+1}$ and $\langle\cdot, \cdot\rangle_{m+1}^{(h)}$. In particular, that scheme would equidistribute a given parameterization after one time step. But the highly nonlinear nature of both of these approximations make them not very practical, in comparison to the fully practical scheme $(2.2 \mathrm{a}, \mathrm{b})$. However, it does not appear possible to prove an analogue of (2.16) for (2.2a,b). Nevertheless, in practice we see that over a number of time steps, the vertices are moved tangentially so that they will eventually be equidistributed. See Section 3 for details.

As our numerical scheme introduces a tangential change to the given parameterization, an interesting question to ask is whether a continuous version of the scheme prescribes a tangential velocity and hence picks one of the family of solutions to (1.2). First, we note from Mikula and Ševčovič (2001, §6) that if

$$
\vec{x}_{t}=\mathcal{V} \vec{\nu}+\psi \vec{\tau}
$$

then it is easily deduced that

$$
\left(\frac{\left|\vec{x}_{\rho}(\rho, t)\right|}{|\Gamma(t)|}\right)_{t}=0 \quad \forall \rho \in J, \text { and } t>0 \quad \Longleftrightarrow \quad \psi_{\rho}=\varkappa \mathcal{V}\left|\vec{x}_{\rho}\right|-\frac{\left|\vec{x}_{\rho}\right|}{|\Gamma(t)|} \int_{\Gamma(t)} \varkappa \mathcal{V} \mathrm{d} s
$$

that is, the relative local length is preserved for, and only for, a tangential velocity fulfilling the above condition. Assuming that $\vec{X}^{0}$ is equidistributed, then one can show that the scheme $(2.14 \mathrm{a}, \mathrm{b})$ approximates $(2.17)$ with $\mathcal{V}=-\Delta_{s} \varkappa$. Since, on choosing $\vec{\eta}=\left(\vec{X}_{j}\right)_{t} \phi_{j}$ in $(2.14 \mathrm{~b})$ it follows for all $t>0$ that

$$
\begin{aligned}
& \quad\left|\vec{X}_{j}-\vec{X}_{j-1}\right|_{t}=\Psi_{j}-\Psi_{j-1}-\left\langle\kappa \vec{\nu}^{h}, \vec{X}_{t} \phi_{j}\right\rangle^{h} \quad j=1 \rightarrow N, \\
& \text { where } \quad \Psi_{j}=\frac{\vec{X}_{j+1}-\vec{X}_{j}}{\left|\vec{X}_{j+1}-\vec{X}_{j}\right|} \cdot\left(\vec{X}_{j}\right)_{t}
\end{aligned}
$$

approximates the tangential velocity at node $j$. Summing (2.19a) over $j$, and noting the periodicity, yields that

$$
\left|\Gamma^{h}\right|_{t}=-\left\langle\kappa \vec{\nu}^{h}, \vec{X}_{t}\right\rangle^{h} .
$$

Assuming for all $j$ and for all $t>0$ that $\left(\vec{X}_{j+1}-\vec{X}_{j}\right) \nVdash\left(\vec{X}_{j}-\vec{X}_{j-1}\right)$, then (2.16) yields for all $t>0$ and for $j=1 \rightarrow N$ that

$$
\left(\frac{\left|\vec{X}_{j}-\vec{X}_{j-1}\right|}{\left|\Gamma^{h}\right|}\right)_{t}=0 \Longleftrightarrow\left|\vec{X}_{j}-\vec{X}_{j-1}\right|_{t}=\frac{\left|\vec{X}_{j}-\vec{X}_{j-1}\right|}{\left|\Gamma^{h}\right|}\left|\Gamma^{h}\right|_{t} .
$$

Hence it follows from (2.21), (2.19a,b) and (2.20) for all $t>0$ and for $j=1 \rightarrow N$ that

$$
\Psi_{j}-\Psi_{j-1}=\left\langle\kappa \vec{\nu}^{h}, \vec{X}_{t} \phi_{j}\right\rangle^{h}-\frac{\left|\vec{X}_{j}-\vec{X}_{j-1}\right|}{\left|\Gamma^{h}\right|}\left\langle\kappa \vec{\nu}^{h}, \vec{X}_{t}\right\rangle^{h}
$$

which approximates (2.18). 
We note that Mikula and Ševčovič (2005, ((4.2)-(4.5)) introduce a complicated scheme, where a tangential force is prescribed explicitly, to achieve equidistribution of nodes. Whereas, our semi-discrete scheme $(2.14 \mathrm{a}, \mathrm{b})$, and a fully implicit version of $(2.2 \mathrm{a}, \mathrm{b})$, achieve this intrinsically. Moreover, in practice the fully practical scheme $(2.2 \mathrm{a}, \mathrm{b})$ moves the nodes tangentially so that they are eventually equidistributed, even if they are not equidistributed initially.

To further illustrate this, we consider a simplified version of our scheme for the approximation of

$$
\mathcal{V}=\vec{x}_{t} \cdot \vec{\nu}=0 .
$$

Similarly to (2.2a,b), the approximation would be: Find $\left\{\vec{X}^{m+1}, \kappa^{m+1}\right\} \in \underline{V}_{0}^{h} \times W_{0}^{h}$ such that

$$
\begin{aligned}
\left\langle\vec{X}^{m+1}-\vec{X}^{m}, \chi \vec{\nu}^{m}\right\rangle_{m}^{h}=0 & \forall \chi \in W_{0}^{h}, \\
\left\langle\kappa^{m+1} \vec{\nu}^{m}, \vec{\eta}\right\rangle_{m}^{h}+\left\langle\nabla_{s} \vec{X}^{m+1}, \nabla_{s} \vec{\eta}\right\rangle_{m}=0 & \forall \vec{\eta} \in \underline{V}_{0}^{h} .
\end{aligned}
$$

Of course, in this case it is possible to eliminate $\kappa^{m+1}$ from $(2.23 \mathrm{a}, \mathrm{b})$. Then one obtains a symmetric positive semi-definite system for the unknown $\beta^{m+1} \in W_{0}^{h}$, where $\vec{X}_{j}^{m+1}=$ $\vec{X}_{j}^{m}+\beta_{j}^{m+1}\left(\vec{\omega}_{j}^{m}\right)^{\perp}, j=1 \rightarrow N$. In particular, it is then straightforward to show that $\vec{X}^{m+1}=\vec{X}^{m}$ solves $(2.23 \mathrm{a}, \mathrm{b})$ if, and only if, $\vec{X}^{m}$ satisfies $(2.16)$ for all $j$; that is, $\vec{X}^{m}$ is either equidistributed or locally parallel. Moreover, in practice we observe that the scheme $(2.23 \mathrm{a}, \mathrm{b})$ moves the vertices such that, after a number of steps, they are eventually equidistributed or locally parallel. Note that the natural extensions of the schemes $(2.3 \mathrm{a}, \mathrm{b})$ and $(2.4 \mathrm{a}-\mathrm{d})$ to approximate $(2.22)$ would not change the initial parameterization $\vec{X}^{0}$.

\subsection{Triple junctions}

In this section, we consider the case where a network of curves meeting at triple junction points moves under motion by surface diffusion. Unless otherwise stated, for ease of exposition, from now on we consider the case of three curves $\left(\Gamma_{1}, \Gamma_{2}, \Gamma_{3}\right)$ with surface energies $\sigma:=\left(\sigma_{1}, \sigma_{2}, \sigma_{3}\right)$ meeting at two triple junction points $\Lambda_{1}$ and $\Lambda_{2}$, and enclosing two areas, see Figure 2 and in particular note the stated choice of the direction of the unit tangents. We will outline later how the ideas presented for this case can be carried over to the general case, see Remark 2.6 below.

The main idea for the necessary trial (三 test) spaces is to make sure, that the conditions (1.5a-d) hold either essentially or weakly at the triple junctions. Here we will enforce conditions $(1.5 \mathrm{a}, \mathrm{d})$ explicitly through the trial space, whereas conditions $(1.5 \mathrm{~b}, \mathrm{c})$ will be enforced weakly, similarly to a Neumann boundary condition for a standard second order elliptic PDE. In particular, let $I:=[0,1]$ be the unit interval and let

$$
\underline{V}:=\left\{\left(\vec{\chi}_{1}, \vec{\chi}_{2}, \vec{\chi}_{3}\right) \in\left[C\left(I, \mathbb{R}^{2}\right)\right]^{3}: \vec{\chi}_{1}=\vec{\chi}_{2}=\vec{\chi}_{3} \text { on } \partial I\right\}
$$

and

$$
W:=\left\{\left(\chi_{1}, \chi_{2}, \chi_{3}\right) \in[C(I, \mathbb{R})]^{3}: \sigma_{1} \chi_{1}+\sigma_{2} \chi_{2}+\sigma_{3} \chi_{3}=0 \text { on } \partial I\right\}
$$


Given a test function $\vec{\eta} \in \underline{V} \cap\left[H^{1}\left(I, \mathbb{R}^{2}\right)\right]^{3}$, we multiply the second equation in (1.6) on $\Gamma_{i}$ by $\sigma_{i} \eta_{i}$. Integrating over $\Gamma_{i}$ and summing over $i$ yields for the right hand side that

$$
\sum_{i=1}^{3} \sigma_{i} \int_{\Gamma_{i}}\left(\Delta_{s} \vec{x}_{i}\right) \cdot \vec{\eta}_{i} \mathrm{~d} s=-\sum_{i=1}^{3} \sigma_{i} \int_{\Gamma_{i}} \nabla_{s} \vec{x}_{i} \cdot \nabla_{s} \vec{\eta}_{i} \mathrm{~d} s+\sum_{j=1}^{2}(-1)^{j}\left[\sum_{i=1}^{3}\left(\left.\sigma_{i} \vec{\tau}_{i}\right|_{\Lambda_{j}}\right) \cdot \vec{\eta}_{i}\left(\Lambda_{j}\right)\right],
$$

where we have used integration by parts and observed the fact that $\nabla_{s} \vec{x}_{i}=\vec{\tau}_{i} \vec{\tau}_{i}^{T}$. Clearly, dropping the last term in (2.24) corresponds, as $\vec{\eta} \in \underline{V}$, to weakly enforcing Young's law (1.5b) at the triple junction points $\Lambda_{1}$ and $\Lambda_{2}$. Similarly, testing the first equation in (1.6) with a function $\chi \in W$ yields a weak approximation of $(1.5 \mathrm{c})$.

Now let $I=\cup_{j=1}^{N_{i}} I_{j}^{i}, i=1 \rightarrow 3$, be decompositions of $I$ into intervals $I_{j}^{i}=\left[q_{j-1}^{i}, q_{j}^{i}\right]$ based on the nodes $\left\{q_{j}^{i}\right\}_{j=0}^{N_{i}}, N_{i} \geq 2$. Let $h_{j}^{i}=\left|I_{j}^{i}\right|$ and $h=\max _{i=1 \rightarrow 3} \max _{j=1 \rightarrow N_{i}} h_{j}^{i}$ be the maximal length of a grid element. The appropriate finite element spaces are then defined by

$$
\underline{V}^{h}:=\left\{\left(\vec{\chi}_{1}, \vec{\chi}_{2}, \vec{\chi}_{3}\right) \in \underline{V}:\left.\vec{\chi}_{i}\right|_{I_{j}^{i}} \text { is linear } \forall j=1 \rightarrow N_{i}, i=1 \rightarrow 3\right\} \subset \underline{V}
$$

and similarly for the space of scalar functions $W^{h} \subset W$.

Recall the time partitioning $\left\{\tau_{m}\right\}_{m=0}^{M-1}$ and let $\vec{X}^{m} \in \underline{V}^{h}$ be an approximation to $\vec{x}\left(\cdot, t_{m}\right) \equiv\left(\vec{x}_{1}, \vec{x}_{2}, \vec{x}_{3}\right)\left(\cdot, t_{m}\right)$, and similarly $\kappa^{m} \in W^{h}$ for $\varkappa\left(\cdot, t_{m}\right)$. We introduce the $\sigma$ weighted $L^{2}$ inner product $\langle\cdot, \cdot\rangle_{m}$, and its mass lumped analogue $\langle\cdot, \cdot\rangle_{m}^{h}$, over the current surface $\Gamma^{m}:=\left(\Gamma_{1}^{m}, \Gamma_{2}^{m}, \Gamma_{3}^{m}\right)$, which is described by the vector function $\vec{X}^{m} \in \underline{V}^{h}$, for scalar and vector functions $f, g \in\left[L^{2}\left(I, \mathbb{R}^{(2)}\right)\right]^{3}$ as follows:

$$
\begin{aligned}
\langle f, g\rangle_{m} & :=\int_{\Gamma^{m}} f \cdot g \mathrm{~d} s:=\sum_{i=1}^{3} \sigma_{i} \int_{I} f_{i} \cdot g_{i}\left|\left(\vec{X}_{i}^{m}\right)_{\rho}\right| \mathrm{d} \rho \\
\langle f, g\rangle_{m}^{h} & :=\sum_{i=1}^{3} \frac{\sigma_{i}}{2} \sum_{j=1}^{N_{i}}\left|\vec{X}_{i}^{m}\left(q_{j}^{i}\right)-\vec{X}_{i}^{m}\left(q_{j-1}^{i}\right)\right|\left[\left(f_{i} \cdot g_{i}\right)\left(\left[q_{j}^{i}\right]^{-}\right)+\left(f_{i} \cdot g_{i}\right)\left(\left[q_{j-1}^{i}\right]^{+}\right)\right] .
\end{aligned}
$$

In addition, we note that

$$
\left.\left(\nabla_{s} f . \nabla_{s} g\right)\right|_{\Gamma_{i}^{m}}=\frac{\left(f_{i}\right)_{\rho} \cdot\left(g_{i}\right)_{\rho}}{\left|\left(\vec{X}_{i}^{m}\right)_{\rho}\right|^{2}}, \quad \vec{\nu}^{m} \mid \Gamma_{i}^{m}=-\frac{\left[\left(\vec{X}_{i}^{m}\right)_{\rho}\right]^{\perp}}{\left|\left(\vec{X}_{i}^{m}\right)_{\rho}\right|}, \quad i=1 \rightarrow 3 .
$$

Using a weak formulation of (1.6) and (1.5a-d) as indicated in (2.24), we then propose the following approximation to (1.6) and (1.5a-d): Find $\left\{\vec{X}^{m+1}, \kappa^{m+1}\right\} \in \underline{V}^{h} \times W^{h}$ such that

$$
\begin{array}{rr}
\left\langle\frac{\vec{X}^{m+1}-\vec{X}^{m}}{\tau_{m}}, \chi \vec{\nu}^{m}\right\rangle_{m}^{h}-\left\langle\sigma \nabla_{s} \kappa^{m+1}, \nabla_{s} \chi\right\rangle_{m}=0 & \forall \chi \in W^{h}, \\
\left\langle\kappa^{m+1} \vec{\nu}^{m}, \vec{\eta}\right\rangle_{m}^{h}+\left\langle\nabla_{s} \vec{X}^{m+1}, \nabla_{s} \vec{\eta}\right\rangle_{m}=0 & \forall \vec{\eta} \in \underline{V}^{h} .
\end{array}
$$

Before we can proceed to prove existence and uniqueness to $(2.26 \mathrm{a}, \mathrm{b})$, we have to make the following very mild assumption. 
$(\mathcal{A})$ Let $\left|\left(\vec{X}_{i}^{m}\right)_{\rho}\right|>0$ for almost all $\rho \in I, i=1 \rightarrow 3$. Let $\vec{\nu}_{i, j-\frac{1}{2}}^{m}:=-\left.\frac{\left[\left(\vec{X}_{i}^{m}\right)_{\rho}\right]^{\perp}}{\left|\left(\vec{X}_{i}^{m}\right)_{\rho}\right|}\right|_{I_{j}^{i}}, j=$ $1 \rightarrow N_{i}$ and set $\vec{\omega}_{i, j}^{m}:=\frac{\left|\vec{X}_{i}^{m}\left(q_{j}^{i}\right)-\vec{X}_{i}^{m}\left(q_{j-1}^{i}\right)\right| \vec{\nu}_{i, j-\frac{1}{2}}^{m}+\left|\vec{X}_{i}^{m}\left(q_{j+1}^{i}\right)-\vec{X}_{i}^{m}\left(q_{j}^{i}\right)\right| \vec{\nu}_{i, j+\frac{1}{2}}^{m}}{\left|\vec{X}_{i}^{m}\left(q_{j}^{i}\right)-\vec{X}_{i}^{m}\left(q_{j-1}^{i}\right)\right|+\left|\vec{X}_{i}^{m}\left(q_{j+1}^{i}\right)-\vec{X}_{i}^{m}\left(q_{j}^{i}\right)\right|}, j=1 \rightarrow N_{i}-1$, $i=1 \rightarrow 3$. Then we assume further that for each $i=1 \rightarrow 3$ there exists a $j \in$ $\left\{1, \ldots, N_{i}-1\right\}$ such that $\vec{\omega}_{i, j}^{m} \neq \overrightarrow{0}$. Moreover, we require that $\operatorname{dim} \operatorname{span}\left\{\left\{\vec{\omega}_{i, j}^{m}\right\}_{j=1}^{N_{i}-1}\right\}_{i=1}^{3}$ $=d=2$.

The assumption $(\mathcal{A})$ basically assures that none of the curves $\Gamma_{i}^{m}, i=1 \rightarrow 3$, is a "zig zagging" connection between the two triple junctions points $\Lambda_{1}$ and $\Lambda_{2}$. A sufficient condition for $(\mathcal{A})$ to hold is that at least one of the three curves is not a "saw tooth" like curve similarly to the one in Figure 3, where all the vertex normals $\vec{\omega}_{i, j}^{m}, j=1 \rightarrow N_{i}-1$, are linearly dependent.

TheOREM. 2.2. Let the assumption $(\mathcal{A})$ hold. Then there exists a unique solution $\left\{\vec{X}^{m+1}, \kappa^{m+1}\right\} \in \underline{V}^{h} \times W^{h}$ to the system $(2.26 \mathrm{a}, \mathrm{b})$.

Proof. As $(2.26 \mathrm{a}, \mathrm{b})$ is linear, existence follows from uniqueness. To investigate the latter, we consider the system: Find $\{\vec{X}, \kappa\} \in \underline{V}^{h} \times W^{h}$ such that

$$
\begin{array}{rlrl}
\left\langle\vec{X}, \chi \vec{\nu}^{m}\right\rangle_{m}^{h}-\tau_{m}\left\langle\sigma \nabla_{s} \kappa, \nabla_{s} \chi\right\rangle_{m} & =0 & & \forall \chi \in W^{h} \\
\left\langle\kappa \vec{\nu}^{m}, \vec{\eta}\right\rangle_{m}^{h}+\left\langle\nabla_{s} \vec{X}, \nabla_{s} \vec{\eta}\right\rangle_{m} & =0 & \forall \vec{\eta} \in \underline{V}^{h} .
\end{array}
$$

Similarly to (2.6a,b), choosing $\chi=\kappa \in W^{h}$ in (2.27a) and $\vec{\eta}=\vec{X} \in \underline{V}^{h}$ in (2.27b) yields that

$$
\left\langle\nabla_{s} \vec{X}, \nabla_{s} \vec{X}\right\rangle_{m}+\tau_{m}\left\langle\sigma \nabla_{s} \kappa, \nabla_{s} \kappa\right\rangle_{m}=0
$$

It follows from (2.28) that $\kappa \equiv \kappa^{c}=\left(\kappa_{1}^{c}, \kappa_{2}^{c}, \kappa_{3}^{c}\right)^{T} \in \mathbb{R}^{3}$ such that $\sum_{i=1}^{3} \sigma_{i} \kappa_{i}^{c}=0$ and $\vec{X} \equiv \vec{X}^{c}=\left(\vec{X}_{1}^{c}, \vec{X}_{2}^{c}, \vec{X}_{3}^{c}\right)^{T} \in\left(\mathbb{R}^{2}\right)^{3}$ with $\vec{X}_{1}^{c}=\vec{X}_{2}^{c}=\vec{X}_{3}^{c}$; and hence

$$
\left\langle\vec{X}^{c}, \chi \vec{\nu}^{m}\right\rangle_{m}^{h}=0 \quad \forall \chi \in W^{h}, \quad\left\langle\kappa^{c} \vec{\nu}^{m}, \vec{\eta}_{m}^{h}=0 \quad \forall \vec{\eta} \in \underline{V}^{h} .\right.
$$

Similarly to (2.9), choosing $\vec{\eta}=\vec{z} \varphi_{j}^{i} \in \underline{V}^{h}$, for a fixed $i=1 \rightarrow 3$ and $j=1 \rightarrow N_{i}-1$ in (2.29), with $\varphi_{j}^{i} \in W^{h}$ being the standard basis function associated with $q_{j}^{i}$, and noting (2.25) yields, on assuming $\kappa_{i}^{c} \neq 0$, that for all $j=1 \rightarrow N_{i}-1$

$$
\vec{\omega}_{i, j}^{m} \cdot \vec{z}=0 \quad \forall \vec{z} \in \mathbb{R}^{2} \quad \Longleftrightarrow \quad \vec{\omega}_{i, j}^{m}=\overrightarrow{0}
$$

However, this contradicts assumption $(\mathcal{A})$ and hence $\kappa_{i}^{c}=0, i=1 \rightarrow 3$. Similarly, choosing $\chi=\varphi_{j}^{i}$ in $(2.29)$ and noting that $\vec{X}_{1}^{c}=\vec{X}_{2}^{c}=\vec{X}_{3}^{c}$ yields that

$$
\vec{X}_{1}^{c} \cdot \vec{\omega}_{i, j}^{m}=0 \quad \forall j=1 \rightarrow N_{i}-1, i=1 \rightarrow 3
$$

Assumption $(\mathcal{A})$ then yields that $\vec{X}_{1}^{c}=\overrightarrow{0}$, and hence $\vec{X}^{c}=\overrightarrow{0}$. Hence we have shown that $(2.26 \mathrm{a}, \mathrm{b})$ has a unique solution $\left\{\vec{X}^{m+1}, \kappa^{m+1}\right\} \in \underline{V}^{h} \times W^{h}$. 
REMARK. 2.5. Similarly to $(2.14 \mathrm{a}, \mathrm{b})$, in a time continuous semidiscrete version of our scheme $(2.26 \mathrm{a}, \mathrm{b})$ we obtain exact area conservation as testing for example with $\chi=$ $\left(-\frac{1}{\sigma_{1}}, \frac{1}{\sigma_{2}}, 0\right) \in W^{h}$ in the analogue of $(2.26 \mathrm{a})$ leads to

$$
0=\int_{\Gamma_{2}^{h}}\left[\vec{X}_{2}\right]_{t} \cdot \vec{\nu}^{h} \mathrm{~d} s-\int_{\Gamma_{1}^{h}}\left[\vec{X}_{1}\right]_{t} \cdot \vec{\nu}^{h} \mathrm{~d} s=\frac{d}{d t} a_{3}^{h}(t)
$$

where $a_{3}^{h}(t)$ is the area enclosed by $\Gamma_{1}^{h}$ and $\Gamma_{2}^{h}$. Moreover, the condition (2.16) now yields that vertices will be equidistributed on all curve segments of $\Gamma_{i}^{h}$, that are not locally parallel. Although we are unable to prove such results for the fully discrete scheme $(2.26 \mathrm{a}, \mathrm{b})$, the change relative to the initial area never exceeded $1 \%$ in our simulations. In addition, we observed the equidistribution property for $(2.26 \mathrm{a}, \mathrm{b})$, see e.g. Figure 15 below.

REMARK. 2.6. The definitions of the spaces $\underline{V}$ and $W$ can easily be generalized to a situation with $K_{B}$ bubbles/areas, $K_{C}$ curves and $K_{T}$ triple junction points; and all the results in this section extend to this general case. Note that the Euler-Poincaré formula yields that $6\left(K_{B}-1\right)=2 K_{C}=3 K_{T}$; e.g. $K_{B}=K_{T}=2$ and $K_{C}=3$ in Figure 2. In particular, we would have that

$$
\begin{aligned}
& \underline{V}:=\left\{\left(\vec{\chi}_{1}, \ldots, \vec{\chi}_{K_{C}}\right) \in\left[C\left(I, \mathbb{R}^{2}\right)\right]^{K_{C}}: \vec{\chi}_{i_{j}}\left(p_{j, i_{j}}\right)=\vec{\chi}_{1_{j}}\left(p_{j, 1_{j}}\right), i=2 \rightarrow 3, \forall j=1 \rightarrow K_{T}\right\}, \\
& W:=\left\{\left(\chi_{1}, \ldots, \chi_{K_{C}}\right) \in[C(I, \mathbb{R})]^{K_{C}}: \sum_{i=1}^{3}(-1)^{p_{j, i_{j}}} \sigma_{i_{j}} \chi_{i_{j}}\left(p_{j, i_{j}}\right)=0 \quad \forall j=1 \rightarrow K_{T}\right\} .
\end{aligned}
$$

Here $i_{j} \in\left\{1, \ldots, K_{C}\right\}, i=1 \rightarrow 3$, denotes the 3 curves meeting at triple junction $j$, while $p_{j, i_{j}} \in\{0,1\}$ denotes whether these curves start $\left(p_{j, i_{j}}=0\right)$ or end $\left(p_{j, i_{j}}=1\right)$ at the triple junction point $j$. I.e. $\left|\left\{i_{j}: i=1 \rightarrow 3\right\}\right|=3$ for all $j=1 \rightarrow K_{T},\left|\left\{j: i_{j}=c\right\}\right|=2$ for all $c=1 \rightarrow K_{C}$ and $\sum_{j=1}^{K_{T}} p_{j, c}=1$ for all $c=1 \rightarrow K_{C}$.

Furthermore, we can establish that our scheme is unconditionally stable.

Theorem. 2.3. Let $\left\{\vec{X}^{m}, \kappa^{m}\right\}_{m=1}^{M}$ be the solution of $(2.26 \mathrm{a}, \mathrm{b})$. Then for all $k=1 \rightarrow M$ we have

$$
\left|\Gamma^{k}\right|+\sum_{m=0}^{k-1} \tau_{m} \int_{\Gamma^{m}} \sigma\left|\nabla_{s} \kappa^{m+1}\right|^{2} \mathrm{~d} s \leq\left|\Gamma^{0}\right|,
$$

where $\left|\Gamma^{k}\right|:=\int_{\Gamma^{k}} 1 \mathrm{~d} s \equiv \sum_{i=1}^{3} \sigma_{i}\left|\Gamma_{i}^{k}\right|$ on recalling the definition (2.25). that

Proof. Choosing $\chi=\kappa^{m+1} \in W^{h}$ in (2.26a) and $\vec{\eta}=\frac{\vec{X}^{m+1}-\vec{X}^{m}}{\tau_{m}} \in \underline{V}^{h}$ in (2.26b) yields

$$
\left\langle\nabla_{s} \vec{X}^{m+1}, \nabla_{s}\left(\vec{X}^{m+1}-\vec{X}^{m}\right)\right\rangle_{m}+\tau_{m}\left\langle\sigma \nabla_{s} \kappa^{m+1}, \nabla_{s} \kappa^{m+1}\right\rangle_{m}=0 .
$$

We now analyse the first term in (2.30), using the ideas in Dziuk (1999). Let $\vec{h}_{j}^{i, m}:=$ 
$\vec{X}_{i}^{m}\left(q_{j+1}^{i}\right)-\vec{X}_{i}^{m}\left(q_{j}^{i}\right)$. Then it holds that

$$
\begin{aligned}
& \left\langle\nabla_{s} \vec{X}^{m+1}, \nabla_{s}\left(\vec{X}^{m+1}-\vec{X}^{m}\right)\right\rangle_{m}=\sum_{i=1}^{3} \sigma_{i} \int_{\Gamma_{i}^{m}}\left[\nabla_{s} \vec{X}^{m+1}\right] \cdot\left[\nabla_{s}\left(\vec{X}^{m+1}-\vec{X}^{m}\right)\right] \mathrm{d} s \\
& \quad=\sum_{i=1}^{3} \sigma_{i} \sum_{j=1}^{N_{i}-1}\left[\frac{\left|\vec{h}_{j}^{i, m+1}\right|^{2}-\vec{h}_{j}^{i, m+1} \cdot \vec{h}_{j}^{i, m}}{\left|\vec{h}_{j}^{i, m}\right|}\right] \\
& \quad=\sum_{i=1}^{3} \sigma_{i} \sum_{j=1}^{N_{i}-1}\left[\frac{\left(\left|\vec{h}_{j}^{i, m+1}\right|-\left|\vec{h}_{j}^{i, m}\right|\right)^{2}+\left|\vec{h}_{j}^{i, m+1}\right|\left|\vec{h}_{j}^{i, m}\right|-\vec{h}_{j}^{i, m+1} \cdot \vec{h}_{j}^{i, m}}{\left|\vec{h}_{j}^{i, m}\right|}+\left|\vec{h}_{j}^{i, m+1}\right|-\left|\vec{h}_{j}^{i, m}\right|\right] \\
& \quad \geq \sum_{i=1}^{3} \sigma_{i} \sum_{j=1}^{N_{i}-1}\left[\left|\vec{h}_{j}^{i, m+1}\right|-\left|\vec{h}_{j}^{i, m}\right|\right]=\left|\Gamma^{m+1}\right|-\left|\Gamma^{m}\right| .
\end{aligned}
$$

Combining (2.30) and (2.31) yields that

$$
\left|\Gamma^{m+1}\right|-\left|\Gamma^{m}\right|+\tau_{m} \int_{\Gamma^{m}} \sigma\left|\nabla_{s} \kappa^{m+1}\right|^{2} \mathrm{~d} s \leq 0 .
$$

Summing (2.32) for $m=0 \rightarrow k-1$ yields the desired result.

The proof above is written explicitly for $(2.26 \mathrm{a}, \mathrm{b})$ but as it depends solely on a specific choice of test functions it immediately carries over to $(2.2 \mathrm{a}, \mathrm{b})$.

We now extend the Schur complement approach developed above for closed curves to triple junctions. Let $N:=\sum_{i=1}^{3}\left(N_{i}+1\right)$. We define the orthogonal projections

$$
K: \mathbb{R}^{N} \rightarrow \mathbb{X}:=\left\{\left(z_{1}, z_{2}, z_{3}\right) \in \mathbb{R}^{N}: \sum_{i=1}^{3} \sigma_{i}\left[z_{i}\right]_{0}=\sum_{i=1}^{3} \sigma_{i}\left[z_{i}\right]_{N_{i}}=0\right\}
$$

and $\vec{P}:\left(\mathbb{R}^{2}\right)^{N} \rightarrow \underline{\mathbb{X}}:=\left\{\left(\vec{z}_{1}, \vec{z}_{2}, \vec{z}_{3}\right) \in\left(\mathbb{R}^{2}\right)^{N}:\left[\vec{z}_{1}\right]_{0}=\left[\vec{z}_{2}\right]_{0}=\left[\vec{z}_{3}\right]_{0},\left[\vec{z}_{1}\right]_{N_{1}}=\left[\vec{z}_{2}\right]_{N_{2}}=\left[\vec{z}_{3}\right]_{N_{3}}\right\}$ onto the Euclidean spaces associated with $W^{h}$ and $\underline{V}^{h}$, respectively. These two projections will be crucial for the construction of the linear system for the unknown coefficient vectors in $\mathbb{R}^{N} \times\left(\mathbb{R}^{2}\right)^{N}$ that represent the solution to $(2.26 \mathrm{a}, \mathrm{b})$, rather than having to work with the trial and test spaces $W^{h}$ and $\underline{V}^{h}$ directly. This construction is similar to the standard technique used for an ODE with periodic boundary conditions.

In order to give a matrix formulation for $(2.26 \mathrm{a}, \mathrm{b})$ we introduce the matrices $\vec{N}^{i} \in$ $\left(\mathbb{R}^{2}\right)^{\left(N_{i}+1\right) \times\left(N_{i}+1\right)}, A^{i} \in \mathbb{R}^{\left(N_{i}+1\right) \times\left(N_{i}+1\right)}$ and $\vec{A}^{i} \in\left(\mathbb{R}^{2 \times 2}\right)^{\left(N_{i}+1\right) \times\left(N_{i}+1\right)}, i=1 \rightarrow 3$, defined by

$$
\vec{N}_{k l}^{i}:=\sigma_{i} \int_{\Gamma_{i}^{m}} \pi_{i}^{h}\left[\phi_{k}^{i} \phi_{l}^{i}\right] \vec{\nu}^{m} \mathrm{~d} s, \quad A_{k l}^{i}:=\sigma_{i} \int_{\Gamma_{i}^{m}} \nabla_{s} \phi_{k}^{i} . \nabla_{s} \phi_{l}^{i} \mathrm{~d} s, \quad \vec{A}_{k l}^{i}:=A_{k l}^{i} \overrightarrow{I d}_{1},
$$

where $\left\{\phi_{l}^{i}\right\}_{l=0}^{N_{i}}$ is the standard basis of $S_{i}^{h}:=\left\{\chi \in C(I, \mathbb{R}):\left.\chi\right|_{I_{j}^{i}}\right.$ is linear $\left.\forall j=1 \rightarrow N_{i}\right\}$ and $\pi_{i}^{h}: C(I, \mathbb{R}) \rightarrow S_{i}^{h}$ is the standard interpolation operator at the nodes $\left\{q_{j}^{i}\right\}_{j=0}^{N_{i}}$. Then on introducing the matrices

$$
A:=\left(\begin{array}{ccc}
\sigma_{1} A^{1} & 0 & 0 \\
0 & \sigma_{2} A^{2} & 0 \\
0 & 0 & \sigma_{3} A^{3}
\end{array}\right), \vec{A}:=\left(\begin{array}{ccc}
\vec{A}^{1} & 0 & 0 \\
0 & \vec{A}^{2} & 0 \\
0 & 0 & \vec{A}^{3}
\end{array}\right), \vec{N}:=\left(\begin{array}{ccc}
\vec{N}^{1} & 0 & 0 \\
0 & \vec{N}^{2} & 0 \\
0 & 0 & \vec{N}^{3}
\end{array}\right),
$$


where $A: \mathbb{R}^{N} \rightarrow \mathbb{R}^{N}, \vec{A}:\left(\mathbb{R}^{2}\right)^{N} \rightarrow\left(\mathbb{R}^{2}\right)^{N}$ and $\vec{N}: \mathbb{R}^{N} \rightarrow\left(\mathbb{R}^{2}\right)^{N}$, the system of equations $(2.26 \mathrm{a}, \mathrm{b})$ can be written as: Find $\left\{\delta \vec{X}^{m+1}, \kappa^{m+1}\right\} \in \underline{\mathbb{X}} \times \mathbb{X}$ such that

$$
\left(\begin{array}{cc}
\tau_{m} K A K & -K \vec{N}^{T} \vec{P} \\
\vec{P} \vec{N} K & \vec{P} \vec{A} \vec{P}
\end{array}\right)\left(\begin{array}{c}
\kappa^{m+1} \\
\delta \vec{X}^{m+1}
\end{array}\right)=\left(\begin{array}{c}
0 \\
-\vec{P} \vec{A} \vec{P} \vec{X}^{m}
\end{array}\right) .
$$

Here, with the obvious abuse of notation similarly to $(2.11), \kappa^{m+1}=\left(\kappa_{1}^{m+1}, \kappa_{2}^{m+1}, \kappa_{3}^{m+1}\right)^{T}$ with $\kappa_{i}^{m+1}=\left(\left[\kappa_{i}^{m+1}\right]_{0}, \ldots,\left[\kappa_{i}^{m+1}\right]_{N_{i}}\right), i=1 \rightarrow 3$, and $\delta \vec{X}^{m+1}=\left(\delta \vec{X}_{1}^{m+1}, \delta \vec{X}_{2}^{m+1}, \delta \vec{X}_{3}^{m+1}\right)^{T}$ with $\delta \vec{X}_{i}^{m+1}=\left(\left[\delta \vec{X}_{i}^{m+1}\right]_{0}, \ldots,\left[\delta \vec{X}_{i}^{m+1}\right]_{N_{i}}\right), i=1 \rightarrow 3$, are the vectors of coefficients with respect to the standard basis $\left\{\left\{\phi_{l}^{i}\right\}_{l=0}^{N_{i}}\right\}_{i=1}^{3}$ of $\kappa^{m+1}$ and $\vec{X}^{m+1}-\vec{X}^{m}$ in $(2.26 \mathrm{a}, \mathrm{b})$, respectively.

We note that the kernel of $K A K$ is the direct sum of ker $K=\mathbb{X}^{\perp}$ and the space $E=\operatorname{ker} A \cap \mathbb{X}$ spanned by the two null vectors $e_{1}:=\left(\frac{1}{\sigma_{1}} 1^{1},-\frac{1}{\sigma_{2}} 1^{2}, 0\right) \in \mathbb{X}$ and $e_{2}:=$ $\left(0, \frac{1}{\sigma_{2}} 1^{2},-\frac{1}{\sigma_{3}} 1^{3}\right) \in \mathbb{X}$ of $A$, where $1^{i}:=(1, \ldots, 1)^{T} \in \mathbb{R}^{N_{i}+1}, i=1 \rightarrow 3$. That is, ker $K A K=\mathbb{X}^{\perp} \oplus \operatorname{span}\left\{e_{i}: i=1 \rightarrow 2\right\}$. Introducing the inverse $S$ of $K A K$ restricted on the set $(\operatorname{ker} K A K)^{\perp} \subset \mathbb{R}^{N}$, i.e. $S K A K v=K A K S v=v$ for all $v \in(\operatorname{ker} K A K)^{\perp}$, and defining the space $\mathcal{R}:=\operatorname{span}\left\{\vec{P} \vec{N} K e_{i}: i=1 \rightarrow 2\right\} \equiv\{\vec{P} \vec{N} K v: v \in \operatorname{ker} K A K\} \subset \underline{\mathbb{X}}$; we note from the first equation of (2.35) that $\delta \vec{X}^{m+1} \in \mathcal{T}:=\mathcal{R}^{\perp} \cap \underline{\mathbb{X}}$ and hence that $K \vec{N}^{T} \vec{P} \delta \vec{X}^{m+1} \in(\text { ker } K A K)^{\perp}$. Therefore, we can employ a Schur complement approach in order to transform $(2.35)$ to

$$
\begin{aligned}
\kappa^{m+1} & =\frac{1}{\tau_{m}} S K \vec{N}^{T} \vec{P} \delta \vec{X}^{m+1}+\sum_{i=1}^{2} \mu_{i} e_{i} \\
\left(\vec{P} \vec{A} \vec{P}+\frac{1}{\tau_{m}} \vec{P} \vec{N} K S K \vec{N}^{T} \vec{P}\right) \delta \vec{X}^{m+1} & =-\vec{P} \vec{A} \vec{P} \vec{X}^{m}-\sum_{i=1}^{2} \mu_{i} \vec{P} \vec{N} K e_{i}, \quad \delta \vec{X}^{m+1} \in \mathcal{T} ;
\end{aligned}
$$

where in $(2.36 \mathrm{a})$ we have used the fact that $\kappa^{m+1} \in \mathbb{X}$ and where $\mu_{i} \in \mathbb{R}$ are unknown. Let $\vec{\Pi}:\left(\mathbb{R}^{2}\right)^{N} \rightarrow \mathcal{R}^{\perp}$ be the orthogonal projection onto $\mathcal{R}^{\perp}$. Then, on noting that $\delta \vec{X}^{m+1} \in \mathcal{R}^{\perp},(2.36 \mathrm{~b})$ can be simplified to

$$
\vec{\Pi} \vec{P}\left(\vec{A}+\frac{1}{\tau_{m}} \vec{N} K S K \vec{N}^{T}\right) \vec{P} \vec{\Pi} \delta \vec{X}^{m+1}=-\vec{\Pi} \vec{P} \vec{A} \vec{P} \vec{X}^{m}
$$

REMARK. 2.7. A possible definition for the projection $\vec{\Pi}$ is $\vec{\Pi}:=\overrightarrow{I d}_{N}-\vec{Q} \vec{Q}^{T}$, where $\operatorname{im} \vec{Q}=\mathcal{R}$ and $\vec{Q}^{T} \vec{Q}=I d_{2}$. I.e. the columns of $\vec{Q} \in\left(\mathbb{R}^{2}\right)^{N \times 2}$ are an orthonormal basis of the subspace $\mathcal{R} \subset\left(\mathbb{R}^{2}\right)^{N}$ spanned by $\vec{P} \vec{N} K e_{i} \equiv \vec{P} \vec{N} e_{i}$, where $e_{i} \in \mathbb{X}, i=1 \rightarrow 2$, are the above mentioned null vectors of $K A K$. We note that the definitions of $\vec{P}$ and $\vec{N}$ yield that $\operatorname{dim} \mathcal{R}=2$. Hence $\vec{\Pi}$ is the orthogonal projection from $\left(\mathbb{R}^{2}\right)^{N}$ onto $(\operatorname{im} \vec{Q})^{\perp} \equiv \mathcal{R}^{\perp}$.

REMARK. 2.8. The definition of $\vec{\Pi}$ can easily be adapted to a situation with $K_{B}$ bubbles/areas. Now the subspace $E$ of the kernel of $K A K$ has dimension $K_{B}$, and a possible basis consists of vectors that each "describe an admissible orientation of the boundary of a bubble" in terms of the given $K_{C}$ curves. For example, if $K_{B}=3$ and one area is enclosed 
by curves 1,2,4 and curve 2 is parameterized in the opposite direction to curves 1 and 4, then the corresponding eigenvector would be $\left(\frac{1}{\sigma_{1}} 1^{1},-\frac{1}{\sigma_{2}} 1^{2}, 0, \frac{1}{\sigma_{4}} 1^{4}, 0,0\right)$. A more rigorous justification for this can be found in the Appendix.

ThEOREM. 2.4. Let $\left\{\delta \vec{X}^{m+1}, \kappa^{m+1}\right\} \in \mathbb{X} \times \mathbb{X}$ be the unique solution to (2.35). Then $\delta \vec{X}^{m+1}$ uniquely solves (2.37). Moreover, the operator in (2.37) is symmetric positive definite.

Proof. We have already demonstrated that if $\left\{\delta \vec{X}^{m+1}, \kappa^{m+1}\right\}$ is the unique solution to (2.35), then $\delta \vec{X}^{m+1}$ solves (2.37). It remains to show that the solution $\delta \vec{X}^{m+1}$ to $(2.37)$ forms part of the solution to (2.35). On noting that $\vec{\Pi} \delta \vec{X}^{m+1}=\delta \vec{X}^{m+1}$ it follows from (2.37) that

$$
\vec{P}\left(\vec{A}+\frac{1}{\tau_{m}} \vec{N} K S K \vec{N}^{T}\right) \vec{P} \delta \vec{X}^{m+1}=-\vec{P} \vec{A} \vec{P} \vec{X}^{m}-\sum_{i=1}^{2} \mu_{i} \vec{P} \vec{N} K e_{i}
$$

where $\mu_{i} \in \mathbb{R}$ are uniquely defined by

$$
\sum_{i=1}^{2} \mu_{i} \vec{P} \vec{N} K e_{i}=\left(\vec{\Pi}-\overrightarrow{I d}_{N}\right)\left[\vec{P} \vec{A} \vec{P} \vec{X}^{m}+\vec{P}\left(\vec{A}+\frac{1}{\tau_{m}} \vec{N} K S K \vec{N}^{T}\right) \vec{P} \delta \vec{X}^{m+1}\right] \in \mathcal{R},
$$

on recalling that the vectors $\left\{\vec{P} \vec{N} K e_{i}\right\}_{i=1}^{2}$ are linearly independent.

On noting that $\delta \vec{X}^{m+1} \in \mathcal{R}^{\perp}$, we have that $K \vec{N}^{T} \vec{P} \delta \vec{X}^{m+1} \in(\operatorname{ker} K A K)^{\perp}$, and hence we can define $\kappa^{m+1} \in \mathbb{X}$ uniquely by

$$
\kappa^{m+1}=\tau_{m}^{-1} S K \vec{N}^{T} \vec{P} \delta \vec{X}^{m+1}+\sum_{i=1}^{2} \mu_{i} e_{i} .
$$

Combining (2.38) and (2.39) gives that

$$
\vec{P} \vec{A} \vec{P} \delta \vec{X}^{m+1}+\vec{P} \vec{N} K \kappa^{m+1}=-\vec{P} \vec{A} \vec{P} \vec{X}^{m},
$$

while multiplying (2.39) with $K A K$ yields that

$$
\tau_{m} K A K \kappa^{m+1}=K \vec{N}^{T} \vec{P} \delta \vec{X}^{m+1} .
$$

Combining (2.40) and (2.41) yields that $\left\{\delta \vec{X}^{m+1}, \kappa^{m+1}\right\} \in \underline{\mathbb{X}} \times \mathbb{X}$ is the unique solution to (2.35). Moreover, $\delta \vec{X}^{m+1}$ is the unique solution to (2.37).

Finally, on recalling the definition of $\mathcal{T}$, we note that $\vec{\Pi} \mathcal{T} \subset \mathcal{R}^{\perp}$ and $K \vec{N}^{T} \vec{P} \mathcal{R}^{\perp} \subset$ $(\text { ker } K A K)^{\perp}$. Since the operator $K A K$ is symmetric and positive definite on $(\text { ker } K A K)^{\perp}$, it is easily established that $\vec{\Pi} \vec{P} \vec{N} K S K \vec{N}^{T} \vec{P} \vec{\Pi}: \mathcal{T} \rightarrow \mathcal{T}$ is symmetric and positive semidefinite. Moreover, the operator $\vec{\Pi} \vec{P} \vec{A} \vec{P} \vec{\Pi}: \mathcal{T} \rightarrow \mathcal{T}$ is symmetric and positive semidefinite. As (2.37) has a unique solution, the operator in (2.37) is non-singular and hence symmetric positive definite.

The proof above is easily adapted to the closed curve system (2.13). 


\subsection{Willmore flow for curves}

For a closed curve $\Gamma \subset \mathbb{R}^{2}$ and a parameterization $\vec{x} \in \mathbb{R}^{2}$ of $\Gamma$, the system

$$
\vec{x}_{t} \cdot \vec{\nu}=-\Delta_{s} \varkappa-\frac{1}{2} \varkappa^{3}+\lambda \varkappa, \quad \varkappa \vec{\nu}=\Delta_{s} \vec{x}
$$

for $\lambda=0$ models Willmore flow for curves, also called evolution of elastic curves. The inclusion of the given parameter $\lambda \in \mathbb{R}$ either penalizes growth $(\lambda>0)$ or encourages further growth $(\lambda<0)$ in the length of the curve. The time dependent choice

$$
\lambda(t)=\frac{\int_{\Gamma}\left[\frac{1}{2}|\varkappa|^{4}-\left|\nabla_{s} \varkappa\right|^{2}\right] \mathrm{d} s}{\int_{\Gamma}|\varkappa|^{2} \mathrm{~d} s}
$$

models length preserving elastic flow. One can interpret (2.42) as the $L^{2}$ gradient flow of the energy functional

$$
E(\vec{x}):=\int_{\Gamma}\left[\frac{1}{2}|\varkappa|^{2}+\lambda\right] \mathrm{d} s
$$

see e.g. Dziuk, Kuwert, and Schätzle (2002) for details. In fact, for fixed $\lambda \in \mathbb{R}$ testing the first equation in (2.42) with $\vec{x}_{t} \cdot \vec{\nu}$ and integrating over $\Gamma$ yields that

$$
\frac{d}{d t} \int_{\Gamma}\left[\frac{1}{2}|\varkappa|^{2}+\lambda\right] \mathrm{d} s=\int_{\Gamma}\left[\Delta_{s} \varkappa+\frac{1}{2} \varkappa^{3}-\lambda \varkappa\right] \vec{\nu} \cdot \vec{x}_{t} \mathrm{~d} s=-\int_{\Gamma}\left|\vec{x}_{t} \cdot \vec{\nu}\right|^{2} \mathrm{~d} s \leq 0 .
$$

One notes that the energy (2.43) with $\lambda=0$ can be reduced by scaling, as e.g. an expanding circle continuously reduces the energy $E$, and that a parameter $\lambda>0$ acts as a penalization term for growth in the curve's length. For more details on Willmore flow, see also Di Carlo, Gurtin, and Podio-Guidugli (1992), Gurtin and Jabbour (2002), and Deckelnick, Dziuk, and Elliott (2005a).

We can adapt $(2.2 \mathrm{a}, \mathrm{b})$ to include the extra terms as follows: Find $\left\{\vec{X}^{m+1}, \kappa^{m+1}\right\} \in$ $\underline{V}_{0}^{h} \times W_{0}^{h}$ such that

$$
\begin{array}{cc}
\left\langle\frac{\vec{X}^{m+1}-\vec{X}^{m}}{\tau_{m}}, \chi \vec{\nu}^{m}\right\rangle_{m}^{h}-\left\langle\nabla_{s} \kappa^{m+1}, \nabla_{s} \chi\right\rangle_{m}-\left[\lambda_{m}\right]_{+}\left\langle\kappa^{m+1}, \chi\right\rangle_{m}^{h} & \\
=-\frac{1}{2}\left\langle\left(\kappa^{m}\right)^{3}, \chi\right\rangle_{m}^{h}+\left[\lambda_{m}\right]_{-}\left\langle\kappa^{m}, \chi\right\rangle_{m}^{h} & \forall \chi \in W_{0}^{h}, \\
\left\langle\kappa^{m+1} \vec{\nu}^{m}, \vec{\eta}\right\rangle_{m}^{h}+\left\langle\nabla_{s} \vec{X}^{m+1}, \nabla_{s} \vec{\eta}\right\rangle_{m}=0 & \forall \vec{\eta} \in \underline{V}_{0}^{h} ;
\end{array}
$$

where $[r]_{ \pm}:= \pm \max \{ \pm r, 0\}$, and $\kappa^{0} \in W_{0}^{h}$ is suitably chosen, see Section 3 . Note that for a fixed $\lambda \in \mathbb{R}$ we set $\lambda_{m}=\lambda, m=0 \rightarrow M-1$, whereas the time dependent choice

$$
\lambda_{m}=\frac{\frac{1}{2}\left|\left(\kappa^{m}\right)^{2}\right|_{m, h}^{2}-\left|\nabla_{s} \kappa^{m}\right|_{m}^{2}}{\left|\kappa^{m}\right|_{m, h}^{2}},
$$

with $|\cdot|_{m(, h)}^{2}:=\langle\cdot, \cdot\rangle_{m}^{(h)}$, approximates length preserving elastic flow.

TheOREM. 2.5. Let the assumption $\left(\mathcal{A}_{0}\right)$ hold. Then there exists a unique solution $\left\{\vec{X}^{m+1}, \kappa^{m+1}\right\} \in \underline{V}_{0}^{h} \times W_{0}^{h}$ to the system $(2.45 \mathrm{a}, \mathrm{b})$. 
Proof. The proof is the same as the proof of Theorem 2.1, with the only change being the additional term $\tau_{m}\left[\lambda_{m}\right]_{+}\langle\kappa, \kappa\rangle_{m}^{h}$ in $(2.7)$.

Lemma. 2.1. Let $\left\{\vec{X}^{m}, \kappa^{m}\right\}_{m=1}^{M}$ be the solution of $(2.45 \mathrm{a}, \mathrm{b})$. Then, if $\lambda_{m}=\lambda \in \mathbb{R}$ for $m=0 \rightarrow M-1$, we have for all $k=1 \rightarrow M$ that

$$
\begin{aligned}
\left|\Gamma^{k}\right| & +\sum_{m=0}^{k-1} \tau_{m}\left[\left|\nabla_{s} \kappa^{m+1}\right|_{m}^{2}+[\lambda]_{+}\left|\kappa^{m+1}\right|_{m, h}^{2}\right] \\
& \leq\left|\Gamma^{0}\right|+\sum_{m=0}^{k-1} \tau_{m}\left[\frac{1}{2}\left[\frac{3}{4}\left|\left(\kappa^{m}\right)^{2}\right|_{m, h}^{2}+\frac{1}{4}\left|\left(\kappa^{m+1}\right)^{2}\right|_{m, h}^{2}\right]-\frac{[\lambda]_{-}}{2}\left[\left|\kappa^{m}\right|_{m, h}^{2}+\left|\kappa^{m+1}\right|_{m, h}^{2}\right]\right] .
\end{aligned}
$$

Similarly, for the time dependent choice (2.46) we have for all $k=1 \rightarrow M$ that

$$
\left|\Gamma^{k}\right| \leq\left|\Gamma^{0}\right|+\sum_{m=0}^{k-1} \tau_{m} F^{m},
$$

where, for $m=0 \rightarrow M-1$,

$$
\begin{aligned}
F^{m}:= & {\left[\left|\nabla_{s} \kappa^{m}\right|_{m}^{2}-\left|\nabla_{s} \kappa^{m+1}\right|_{m}^{2}\right]+\left(\left[\lambda_{m}\right]_{+}+\frac{1}{2}\left[\lambda_{m}\right]_{-}\right)\left[\left|\kappa^{m}\right|_{m, h}^{2}-\left|\kappa^{m+1}\right|_{m, h}^{2}\right] } \\
& +\frac{1}{8}\left[\left|\left(\kappa^{m+1}\right)^{2}\right|_{m, h}^{2}-\left|\left(\kappa^{m}\right)^{2}\right|_{m, h}^{2}\right] .
\end{aligned}
$$

Proof. One can show similarly to (2.30) that

$$
\begin{aligned}
\frac{1}{\tau_{m}}\left\langle\nabla_{s} \vec{X}^{m+1}, \nabla_{s}\right. & \left.\left(\vec{X}^{m+1}-\vec{X}^{m}\right)\right\rangle_{m}+\left\langle\nabla_{s} \kappa^{m+1}, \nabla_{s} \kappa^{m+1}\right\rangle_{m}+\left[\lambda_{m}\right]_{+}\left\langle\kappa^{m+1}, \kappa^{m+1}\right\rangle_{m}^{h} \\
& =\frac{1}{2}\left\langle\left(\kappa^{m}\right)^{3}, \kappa^{m+1}\right\rangle_{m}^{h}-\left[\lambda_{m}\right]_{-}\left\langle\kappa^{m}, \kappa^{m+1}\right\rangle_{m}^{h} \\
& \leq \frac{1}{2}\left[\frac{3}{4}\left|\left(\kappa^{m}\right)^{2}\right|_{m, h}^{2}+\frac{1}{4}\left|\left(\kappa^{m+1}\right)^{2}\right|_{m, h}^{2}\right]-\frac{\left[\lambda_{m}\right]_{-}}{2}\left[\left|\kappa^{m}\right|_{m, h}^{2}+\left|\kappa^{m+1}\right|_{m, h}^{2}\right]
\end{aligned}
$$

and hence, on noting (2.31), that

$$
\begin{aligned}
\frac{1}{\tau_{m}}\left(\left|\Gamma^{m+1}\right|-\left|\Gamma^{m}\right|\right) & +\left|\nabla_{s} \kappa^{m+1}\right|_{m}^{2}+\left[\lambda_{m}\right]_{+}\left|\kappa^{m+1}\right|_{m, h}^{2} \\
\leq & \frac{1}{2}\left[\frac{3}{4}\left|\left(\kappa^{m}\right)^{2}\right|_{m, h}^{2}+\frac{1}{4}\left|\left(\kappa^{m+1}\right)^{2}\right|_{m, h}^{2}\right]-\frac{\left[\lambda_{m}\right]_{-}}{2}\left[\left|\kappa^{m}\right|_{m, h}^{2}+\left|\kappa^{m+1}\right|_{m, h}^{2}\right] .
\end{aligned}
$$

Summing (2.50) for $m=0 \rightarrow k-1$ yields the desired result (2.47). On the other hand, for the choice (2.46) it follows from the first equality in (2.49) that

$$
\begin{aligned}
\frac{1}{\tau_{m}}\left\langle\nabla_{s} \vec{X}^{m+1},\right. & \left.\nabla_{s}\left(\vec{X}^{m+1}-\vec{X}^{m}\right)\right\rangle_{m}+\left|\nabla_{s} \kappa^{m+1}\right|_{m}^{2}-\left|\nabla_{s} \kappa^{m}\right|_{m}^{2}+\left[\lambda_{m}\right]_{+}\left[\left|\kappa^{m+1}\right|_{m, h}^{2}-\left|\kappa^{m}\right|_{m, h}^{2}\right] \\
& =\frac{1}{2}\left\langle\left(\kappa^{m}\right)^{3}, \kappa^{m+1}-\kappa^{m}\right\rangle_{m}^{h}-\left[\lambda_{m}\right]_{-}\left\langle\kappa^{m}, \kappa^{m+1}-\kappa^{m}\right\rangle_{m}^{h}, \\
& \leq \frac{1}{8}\left[\left|\left(\kappa^{m+1}\right)^{2}\right|_{m, h}^{2}-\left|\left(\kappa^{m}\right)^{2}\right|_{m, h}^{2}\right]-\frac{1}{2}\left[\lambda_{m}\right]_{-}\left[\left|\kappa^{m+1}\right|_{m, h}^{2}-\left|\kappa^{m}\right|_{m, h}^{2}\right] .
\end{aligned}
$$

Combining (2.51) and (2.31) yields, on noting the definition of $F^{m}$, that

$$
\left|\Gamma^{m+1}\right| \leq\left|\Gamma^{m}\right|+\tau_{m} F^{m} .
$$

Summing (2.52) for $m=0 \rightarrow k-1$ yields the desired result (2.48). 
REMARK. 2.9. Clearly a time continuous semidiscrete, or a fully implicit discrete, version of $(2.45 \mathrm{a}, \mathrm{b})$ will inherit the tangential equidistribution property (2.16), as it depends solely on $(2.14 b)$.

In addition, Lemma 2.1 shows that the growth in $\Gamma$ can be controlled by a discrete analogue of $\int_{\Gamma}\left[\frac{1}{2}|\varkappa|^{4}-[\lambda]_{-}|\varkappa|^{2}\right] \mathrm{d} s$. Moreover, on choosing $\chi=\pi^{h}\left[\frac{\vec{X}^{m+1}-\vec{X}^{m}}{\tau_{m}} \cdot \vec{\omega}^{m}\right] \in$ $W_{0}^{h}$ in $(2.45 \mathrm{a})$, where, recall $\left(\mathcal{A}_{0}\right), \vec{\omega}^{m}:=\sum_{j=1}^{N} \vec{\omega}_{j}^{m} \phi_{j} \in \underline{V}_{0}^{h}$; it is straightforward to show that the solution $\left\{\vec{X}^{m+1}, \kappa^{m+1}\right\}$ to $(2.45 \mathrm{a}, \mathrm{b})$ satisfies, e.g. in the case $\lambda=0$,

$$
\begin{aligned}
-\left\langle\nabla_{s} \kappa^{m+1}, \nabla_{s} \pi^{h}\left[\frac{\vec{X}^{m+1}-\vec{X}^{m}}{\tau_{m}} \cdot \vec{\omega}^{m}\right]\right\rangle_{m}+\frac{1}{2}\left\langle\left(\kappa^{m}\right)^{3}, \frac{\vec{X}^{m+1}-\vec{X}^{m}}{\tau_{m}} \cdot \vec{\omega}^{m}\right\rangle_{m}^{h} \\
=-\left\langle\frac{\vec{X}^{m+1}-\vec{X}^{m}}{\tau_{m}},\left[\frac{\vec{X}^{m+1}-\vec{X}^{m}}{\tau_{m}} \cdot \vec{\omega}^{m}\right] \vec{\nu}^{m}\right\rangle_{m}^{h}=-\left|\frac{\vec{X}^{m+1}-\vec{X}^{m}}{\tau_{m}} \cdot \vec{\omega}^{m}\right|_{m, h}^{2}
\end{aligned}
$$

However, it does not seem possible to derive a discrete analogue of (2.44) from (2.53), something that would lead to a discrete scheme that monotonically decreases the Willmore energy (2.43).

Moreover, the bound (2.48) shows that although we cannot guarantee length preservation for our scheme $(2.45 \mathrm{a}, \mathrm{b})$ with the choice (2.46), we can show that the growth in the length of the discrete curve is bounded. In particular, (2.48) suggests that the maximal possible growth goes to zero as $\tau \rightarrow 0$. In fact, in practice the length of the discrete curve is almost exactly preserved, see Section 3.

Similarly to (2.10), we introduce the matrix $M_{0} \in \mathbb{R}^{N \times N}$ by

$$
\left[M_{0}\right]_{k l}:=\left\langle\phi_{k}, \phi_{l}\right\rangle_{m}^{h}=\int_{\Gamma^{m}} \pi^{h}\left[\phi_{k} \phi_{l}\right] \mathrm{d} s
$$

and then rewrite $(2.45 \mathrm{a}, \mathrm{b})$ in terms of

$$
\left(\begin{array}{cc}
\tau_{m}\left(A_{0}+\left[\lambda_{m}\right]_{+} M_{0}\right) & -\vec{N}_{0}^{T} \\
\vec{N}_{0} & \vec{A}_{0}
\end{array}\right)\left(\begin{array}{c}
\kappa^{m+1} \\
\delta \vec{X}^{m+1}
\end{array}\right)=\left(\begin{array}{c}
b \\
-\vec{A}_{0} \vec{X}^{m}
\end{array}\right),
$$

where $b \in \mathbb{R}^{N}$ with $b_{j}=\tau_{m}\left\langle\frac{1}{2}\left(\kappa^{m}\right)^{3}-\left[\lambda_{m}\right]_{-} \kappa^{m}, \phi_{j}\right\rangle_{m}^{h}, j=1 \rightarrow N$.

The solution to $(2.54)$ can be found as follows. If $\lambda_{m}>0$, then $\widehat{A}_{0}:=A_{0}+\left[\lambda_{m}\right]_{+} M_{0}$ is a positive definite matrix, and we can solve (2.54) by solving

$$
\left(\vec{A}_{0}+\frac{1}{\tau_{m}} \vec{N}_{0} \widehat{A}_{0}^{-1} \vec{N}_{0}^{T}\right) \delta \vec{X}^{m+1}=-\vec{A}_{0} \vec{X}^{m}-\frac{1}{\tau_{m}} \vec{N}_{0} \widehat{A}_{0}^{-1} b
$$

and then setting

$$
\kappa^{m+1}=\frac{1}{\tau_{m}} \widehat{A}_{0}^{-1}\left[\vec{N}_{0}^{T} \delta \vec{X}^{m+1}+b\right] .
$$

If $\lambda_{m} \leq 0$, on the other hand, we note that

$$
1^{T}\left(\vec{N}_{0}^{T} \delta \vec{X}^{m+1}+b\right)=0
$$


and, similarly to (2.12a), we have that

$$
\kappa^{m+1}=\frac{1}{\tau_{m}} S_{0}\left[\vec{N}_{0}^{T} \delta \vec{X}^{m+1}+b\right]+\mu 1=\frac{1}{\tau_{m}} S_{0} \Pi\left[\vec{N}_{0}^{T} \delta \vec{X}^{m+1}+b\right]+\mu 1,
$$

where $\Pi=I d_{N}-\frac{11^{T}}{1^{T} 1}$ is the orthogonal projection onto $\left(\operatorname{ker} A_{0}\right)^{\perp}=(\operatorname{span}\{1\})^{\perp}$. Hence $\delta \vec{X}^{m+1}$ satisfies

$$
\left(\vec{A}_{0}+\frac{1}{\tau_{m}} \vec{N}_{0} \Pi S_{0} \Pi \vec{N}_{0}^{T}\right) \delta \vec{X}^{m+1}=-\vec{A}_{0} \vec{X}^{m}-\frac{1}{\tau_{m}} \vec{N}_{0} \Pi S_{0} \Pi b-\mu \vec{N}_{0} 1
$$

Once again, the unique solvability of $(2.45 \mathrm{a}, \mathrm{b})$ yields that the operator $\vec{G}$ on the left hand side of (2.56) is symmetric and positive definite. Hence $\mu$ has to be chosen such that condition (2.55) is satisfied. This can be achieved as follows. Let $\vec{X}_{f}:=\vec{G}^{-1}\left(-\vec{A}_{0} \vec{X}^{m}-\right.$ $\left.\frac{1}{\tau_{m}} \vec{N}_{0} \Pi S_{0} \Pi b\right)$ and $\vec{X}_{g}:=\vec{G}^{-1} \vec{N}_{0} 1$. Then

$$
\mu:=\frac{1^{T} b+1^{T} \vec{N}_{0}^{T} \vec{X}_{f}}{1^{T} \vec{N}_{0}^{T} \vec{X}_{g}} \quad \text { and } \quad \delta \vec{X}^{m+1}=\vec{X}_{f}-\mu \vec{X}_{g} .
$$

Note that $1^{T} \vec{N}_{0}^{T} \vec{X}_{g}=\vec{X}_{g}^{T} \vec{G} \vec{X}_{g}>0$, as $\vec{G}$ is symmetric positive definite, so $\mu$ is well defined and uniquely determined.

\section{Results}

The Schur complement approach (2.37) can be easily solved with a conjugate gradient solver. For the solution of $K A K y=x$ in order to compute $S x$ we can employ an (inner loop) CG solver without a projection, as the right hand side vector $x$ always satisfies the compatibility condition $x \in(\operatorname{ker} K A K)^{\perp}$. See Hestenes (1975) for a justification of using a CG solver for a positive semi-definite system.

We note that the implementation of the orthogonal projection (2.33) is straightforward. Let $w^{k}=\left(w_{1}^{k}, w_{2}^{k}, w_{3}^{k}\right) \in \mathbb{R}^{N}, k=0 \rightarrow 1$, be defined by $\left[w_{i}^{0}\right]_{j}=\sigma_{i} \delta_{0 j}$ and $\left[w_{i}^{1}\right]_{j}=\sigma_{i} \delta_{N_{i} j}, j=0 \rightarrow N_{i}, i=1 \rightarrow 3$. It then holds that $K=K_{0} K_{1}$ with $K_{k}:=I d_{N}-\frac{w^{k}\left[w^{k}\right]^{T}}{\left[w^{k}\right]^{T} w^{k}}, k=0 \rightarrow 1$. The projection $\vec{P}$ can be constructed in a similar way.

Throughout this section we use uniform time steps $\tau_{m}=\tau, m=0 \rightarrow M-1$. For later purposes, we define

$$
\vec{X}(t):=\frac{t-t_{m-1}}{\tau} \vec{X}^{m}+\frac{t_{m}-t}{\tau} \vec{X}^{m-1} \quad t \in\left[t_{m-1}, t_{m}\right] \quad m \geq 1 .
$$

\subsection{Closed curves}

Here we compare the scheme $(2.2 \mathrm{a}, \mathrm{b})$ with two other algorithms in the literature, namely the scheme $(2.3 \mathrm{a}, \mathrm{b})$ from Dziuk, Kuwert, and Schätzle (2002) and the scheme $(2.4 \mathrm{a}-\mathrm{d})$ from Bänsch, Morin, and Nochetto (2005). 

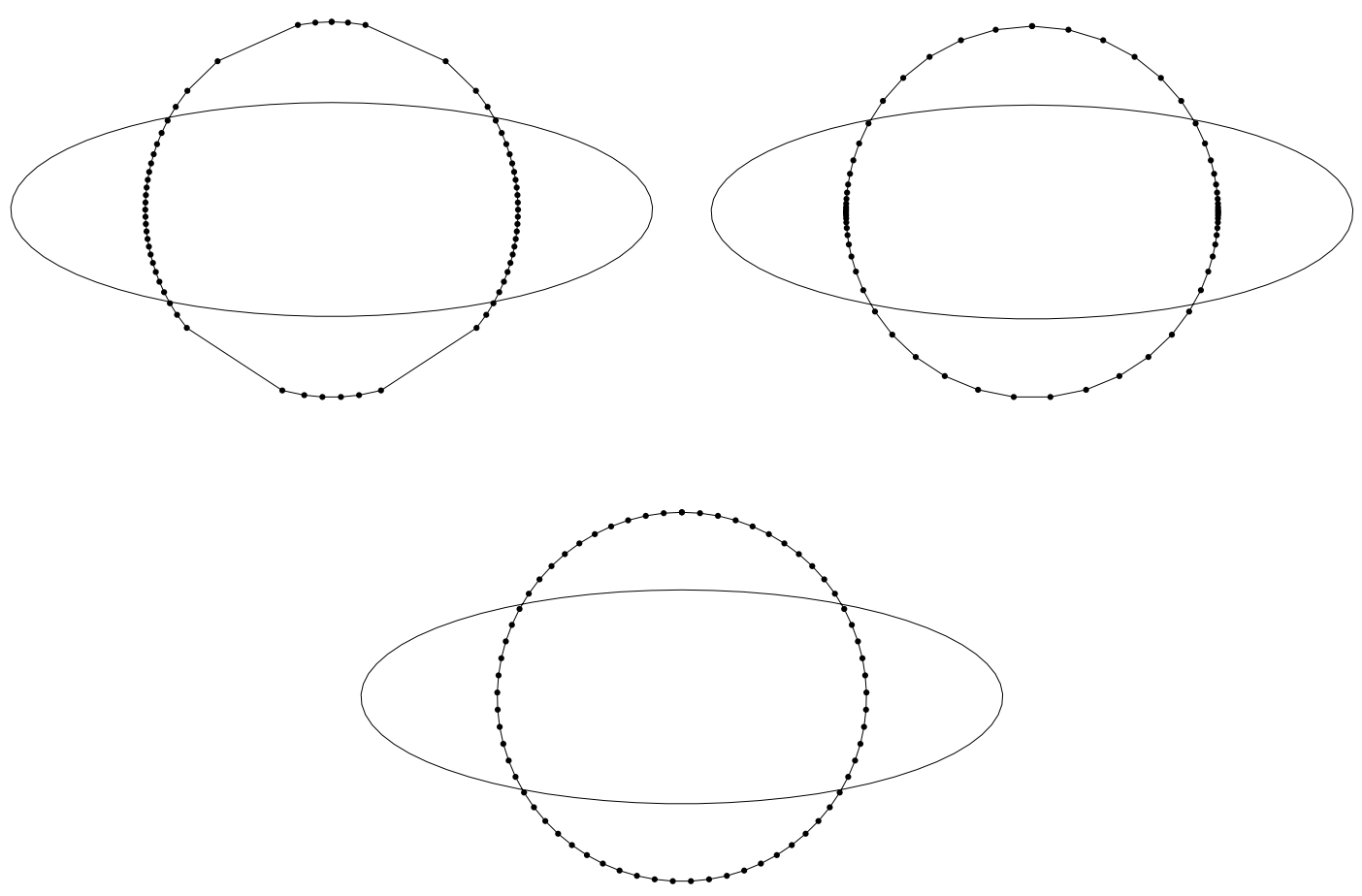

Figure 4: The three schemes for the first experiment, a 3:1 ellipse.

The first experiment is for a mild ellipse. The parameters were chosen as follows. $N=64, \tau=10^{-6}, T=10^{-3}$ and the initial curve is a $3: 1$ ellipse with semiminor axis $R=0.075$. The scheme $(2.3 \mathrm{a}, \mathrm{b})$ eventually breaks down on this experiment, due to the coalescence of grid points. We plot the approximation $\vec{X}$ at time $t=8 \times 10^{-4}$ in Figure 4 (left). Similarly the scheme $(2.4 \mathrm{a}-\mathrm{d})$ breaks down at $t=3.2 \times 10^{-4}$, see Figure 4 (right). We note that a space adaptivity algorithm is described in Bänsch, Morin, and Nochetto (2005) that, while possibly loosing the discrete analogue of (1.3), would most likely be able to integrate further in this example due to the local coarsening of elements. Our scheme $(2.2 \mathrm{a}, \mathrm{b})$, on the other hand, intrinsically moves the vertices such that the problem can be computed until time $t=T$, when the solution has reached the shape of a circle; see the Figure 4 (bottom). Recall that a proof of this is given for the continuous in time semidiscrete scheme in Remark 2.4. Note that the relative area loss for $(2.2 \mathrm{a}, \mathrm{b})$ for this experiment was $0.7 \%$. We remark that no other scheme published in the literature does intrinsically move the mesh points so that no coalescence of mesh points can occur. However, a scheme where a tangential force is prescribed in order to achieve this has been published in Mikula and Ševčovič (2005), recall Remark 2.4. Here a finite difference approximation of a very complicated fourth order system has to be solved, see Mikula and Ševčovič $(2005,(4.2)-(4.5))$.

In order to investigate the three different schemes further, we conduct the following experiment. Starting with an initial curve that consists of a semi-circle and a single additional node on the periphery of the circle, we investigate the ratio $r:=h_{\vec{X}^{m}} / \ell_{\vec{X}^{m}}$, 

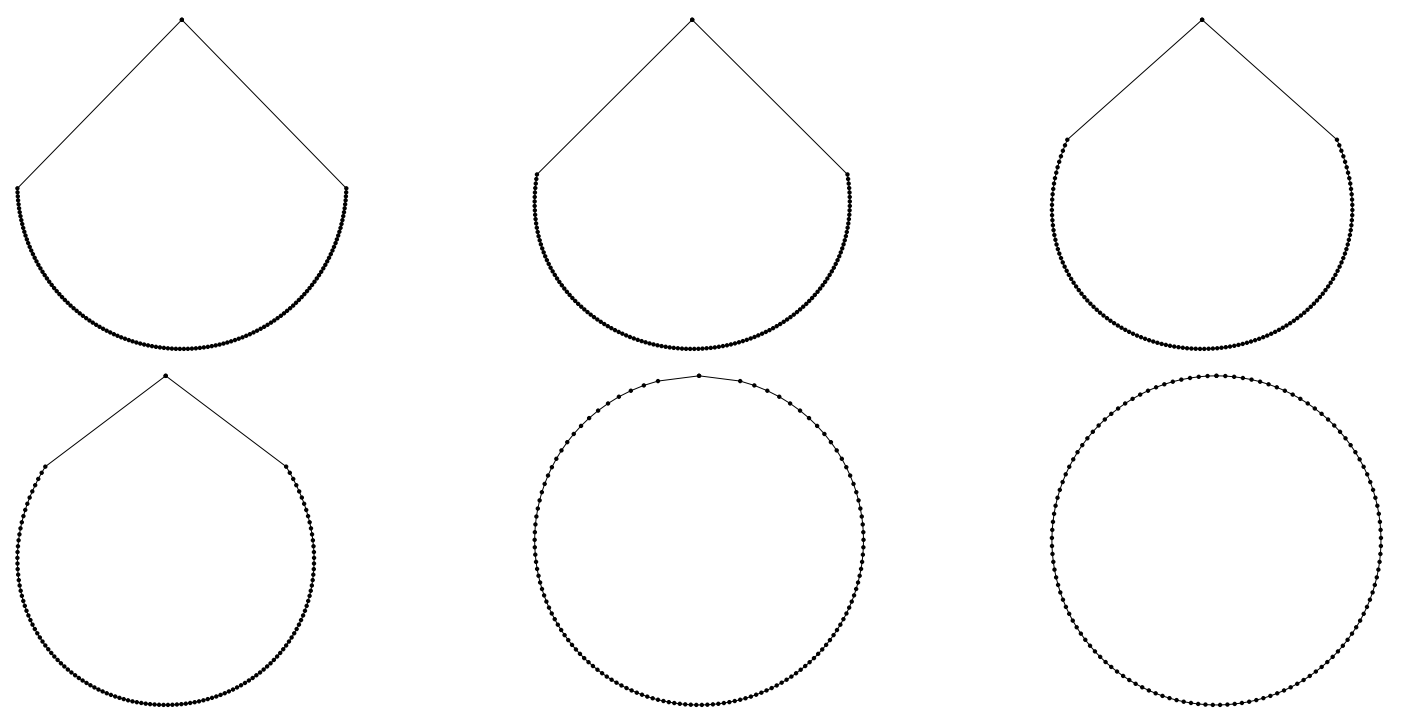

Figure 5: A plot of $\vec{X}$ at times $t=0,10^{-7}, 5 \times 10^{-7}, 10^{-6}, 10^{-5}, T=5 \times 10^{-4}$.

where $h_{\vec{X}^{m}}:=\max _{j=1 \rightarrow N}\left|\vec{X}^{m}\left(q_{j}\right)-\vec{X}^{m}\left(q_{j-1}\right)\right|$ and $\ell_{\vec{X}^{m}}:=\min _{j=1 \rightarrow N}\left|\vec{X}^{m}\left(q_{j}\right)-\vec{X}^{m}\left(q_{j-1}\right)\right|$, over time. We used as parameters $N=128, \tau=10^{-7}, T=5 \times 10^{-4}$ and $R=0.075$ as the radius of the circle. The evolution of $\vec{X}$ for our scheme $(2.2 \mathrm{a}, \mathrm{b})$ can be seen in Figure 5 . Plots of the ratio $r$ for the three schemes can be seen in Figure 6 . We note that scheme $(2.3 \mathrm{a}, \mathrm{b})$ could only compute up to time $t=7.9 \times 10^{-6}$, while scheme $(2.4 \mathrm{a}-\mathrm{d})$ could only compute up to time $t=8.4 \times 10^{-5}$. The last plot in that figure shows the length of the curve computed from our approximation $(2.2 \mathrm{a}, \mathrm{b})$ over time. One can clearly see that although the true solution (a circle) is reached very quickly (at around time $t=2 \times 10^{-5}$ ), in the remaining time the vertices are continually moved tangentially which results in a further decrease in the ratio $r$, which approaches the optimal value 1 .

The coalescence of vertices for the two schemes cited from the literature can be prevented by heuristically redistributing all the mesh points tangentially after each time step, as described in Dziuk, Kuwert, and Schätzle (2002) and Bänsch, Morin, and Nochetto (2005), respectively. In the former case this can lead to excessive loss of area, whereas the latter redistribution is area conserving. To demonstrate the possible effects of these redistributions, we used the same parameters as in the experiment for Figure 4 for an initial $8: 1$ ellipse with semiminor axis $R=0.075$. The results for the three respective schemes can be seen in Figure 7. The relative area loss for our scheme $(2.2 \mathrm{a}, \mathrm{b})$ is $0.7 \%$, while the scheme $(2.3 \mathrm{a}, \mathrm{b})$ (now with a redistribution after each time step) lost $14.1 \%$ of the area. The scheme $(2.4 \mathrm{a}-\mathrm{d})$ (with the redistribution as in Bänsch, Morin, and Nochetto (2005)) lost $-0.7 \%$ of the area. In part, this can have a dramatic effect on the size of the final circular solution.

For a finer mesh, $N=256$, and $\tau=10^{-7}$ we obtained the following results for the $8: 1$ ellipse. For the scheme $(2.3 \mathrm{a}, \mathrm{b})$ with redistribution, the loss of area is $0.8 \%$, while the scheme $(2.4 \mathrm{a}, \mathrm{b})$ with redistribution lost $-0.1 \%$ of the initial area. The scheme $(2.2 \mathrm{a}, \mathrm{b})$ can once again integrate until the final time without artificially redistributing mesh points. 

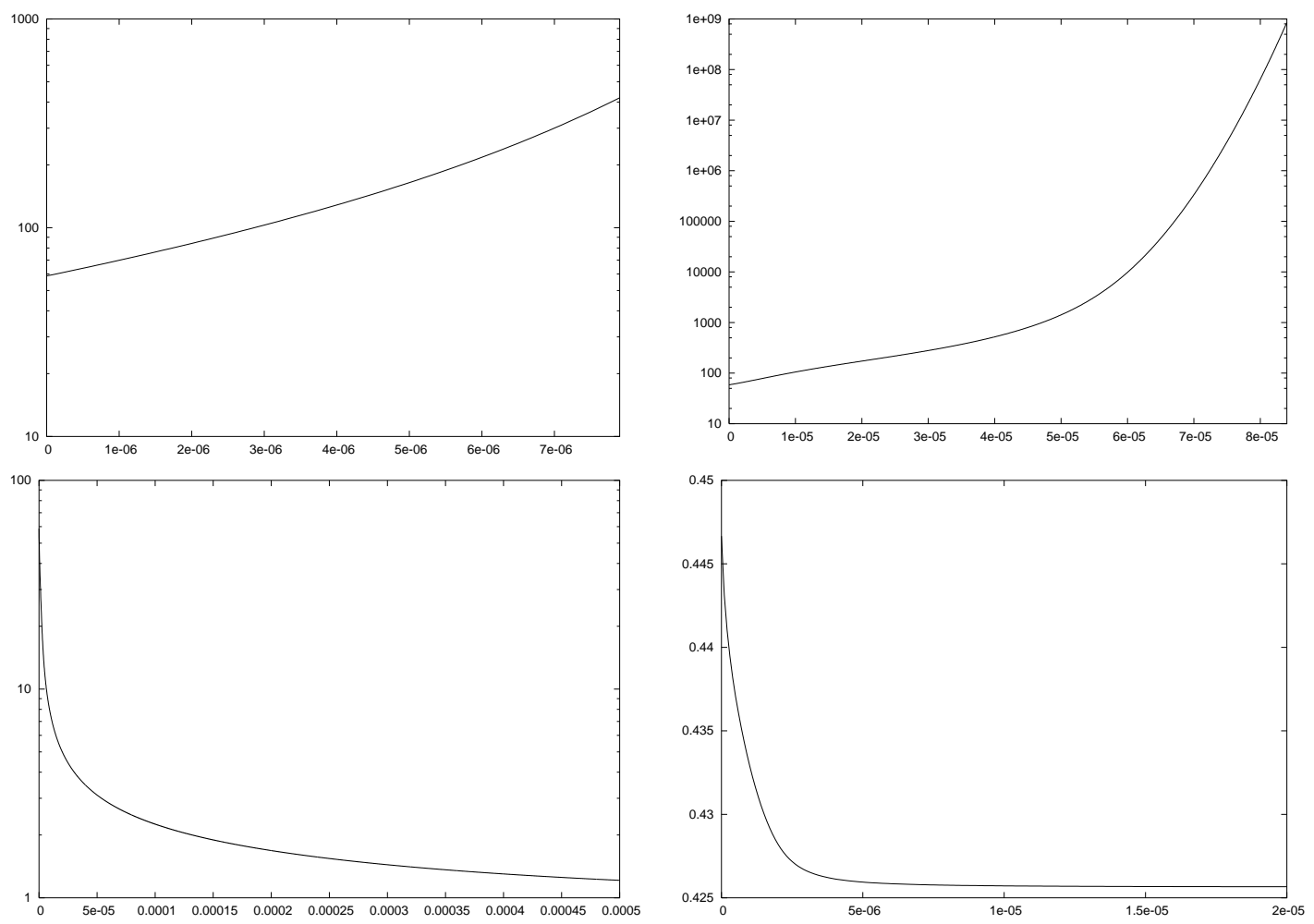

Figure 6: A plot of $\log r$ for the ratio $r=h_{\vec{X}^{m}} / \ell_{\vec{X}^{m}}$ for the three schemes $(2.3 \mathrm{a}, \mathrm{b}),(2.4 \mathrm{a}-\mathrm{d})$ and $(2.2 \mathrm{a}, \mathrm{b})$. The last plot shows the length $|\Gamma(t)|$ for $t \in\left[0,2 \times 10^{-5}\right]$.

The total relative area loss was $0.1 \%$. The final solution can be seen in Figure 8.

In order to demonstrate that our scheme can easily handle examples with sharp corners and concavities, we performed an experiment for the initial curve as in Bänsch, Morin, and Nochetto (2005, Fig. 16), i.e. for a $2 \times 2$ square minus a thin rectangle $(0.02 \times 1.8)$. In Figure 9 we plot $\vec{X}(t)$ for various times $t$ for a computation with discretization parameters $N=256, \tau=10^{-7}$ and $T=2 \times 10^{-3}$. The observed relative area loss was $-0.02 \%$. We note that although the times shown in Figure 9 were chosen close to the ones shown in Bänsch, Morin, and Nochetto (2005), the evolution differs considerably from the one shown there. However, it is difficult to do a direct comparison, as they do not state their discretization parameters. Moreover, as we obtained virtually identical results when
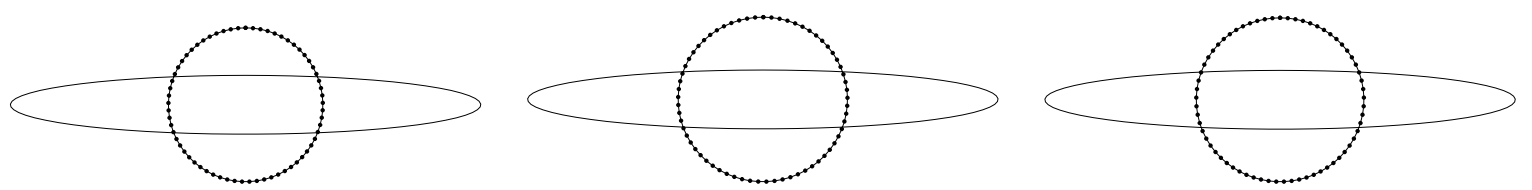

Figure 7: (2.3a,b), (2.4a-d) and (2.2a,b) for the second experiment, an 8:1 ellipse. 


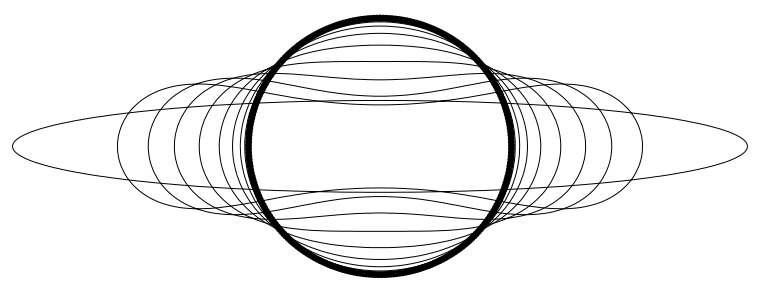

Figure 8: Success for an 8:1 ellipse. Plotted times are $t=0,10^{-4}, 2 \times 10^{-4}, \ldots, 10^{-3}$.

\begin{tabular}{c|c|c}
$k$ & $(2.2 \mathrm{a}, \mathrm{b})$ & $(2.4 \mathrm{a}-\mathrm{d})$ \\
\hline 0 & $5.7897 \mathrm{e}-01$ & $1.0438 \mathrm{e}-01$ \\
1 & $9.2350 \mathrm{e}-02$ & $4.7302 \mathrm{e}-02$ \\
2 & $1.1379 \mathrm{e}-02$ & $7.4513 \mathrm{e}-03$ \\
3 & $1.4182 \mathrm{e}-03$ & $1.0281 \mathrm{e}-03$ \\
4 & $1.0742 \mathrm{e}-04$ & $2.9404 \mathrm{e}-04$ \\
5 & $4.6785 \mathrm{e}-05$ & $2.1847 \mathrm{e}-04$
\end{tabular}

Table 1: Area loss $|a(0)-a(T)|$ for $N=64$ and $\tau=10^{-k}, k=0 \rightarrow 5$.

using our scheme with the coarser discretization parameters $N=128, \tau=10^{-7}$ and $N=256, \tau=10^{-6}$, respectively, we are confident that the results in Figure 9 are accurate. In addition, we include an experiment for a self intersecting curve which was previously computed by Escher, Mayer, and Simonett (1998, Fig. 2), see also Bänsch, Morin, and Nochetto (2005, Fig. 17). The results for the parameters $N=128, \tau=10^{-4}$ and $T=0.075$ can be seen in Figure 10, where we note the excellent qualitative agreement with the other two computations in the literature.

The next experiment underlines the analysis in Remark 2.3. To this end, we fix $N=64, T=2$ and let $\tau=10^{-k}, k=0 \rightarrow 5$ for an initial $2: 1$ ellipse with semiminor axis $R=1$. In Table 1 we report on the area loss, $a^{0}-a^{M}$, for our approximation $(2.2 \mathrm{a}, \mathrm{b})$ as well as for the scheme $(2.4 \mathrm{a}-\mathrm{d})$ from Bänsch, Morin, and Nochetto (2005), which, due to the relatively small curvatures present in the initial curve, could compute the solution without redistributing the nodes. We omit the results for the scheme $(2.3 \mathrm{a}, \mathrm{b})$, as without redistribution of vertices it could only integrate until time $T$ for $k \leq 3$. As is to be expected from the considerations in Remark 2.3 for the semidiscrete approximation $(2.14 \mathrm{a}, \mathrm{b})$, when $\tau \rightarrow 0$ the observed area loss for our scheme $(2.2 \mathrm{a}, \mathrm{b})$ tends to zero. For other schemes, e.g. $(2.4 \mathrm{a}-\mathrm{d})$, we expect the area loss to be bounded from below by some constant depending on $h$.

Finally, we perform the following convergence test. As initial shape we choose an $8: 1$ 

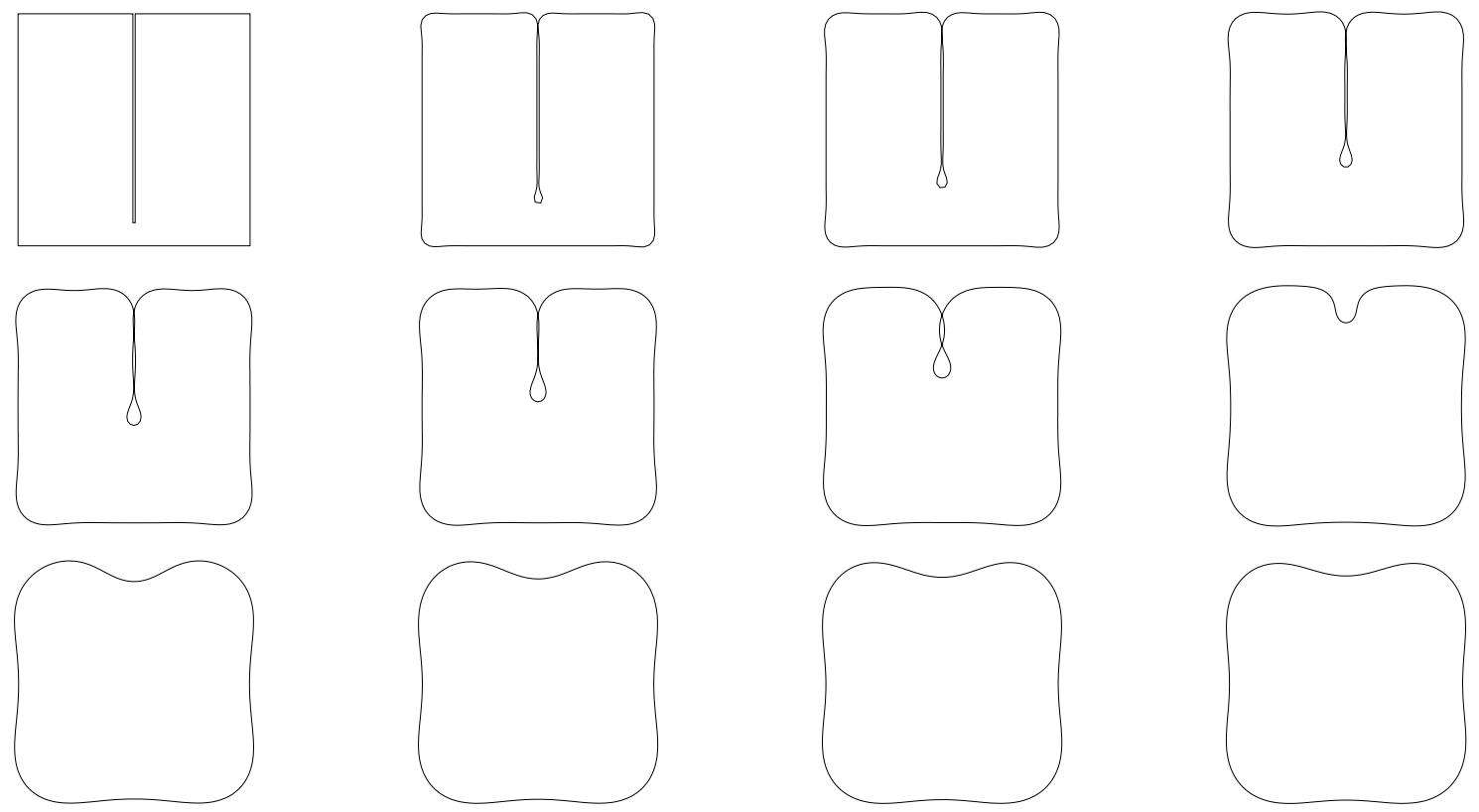

Figure 9: Surface diffusion flow for an almost slit domain. $\vec{X}(t)$ at times $t=0,5 \times$ $10^{-6}, 2 \times 10^{-5}, 6 \times 10^{-5}, 1.2 \times 10^{-4}, 2.3 \times 10^{-4}, 4 \times 10^{4}, 7 \times 10^{-4}, 1.1 \times 10^{-3}, 1.4 \times$ $10^{-3}, 1.7 \times 10^{-3}, T=2 \times 10^{-3}$.
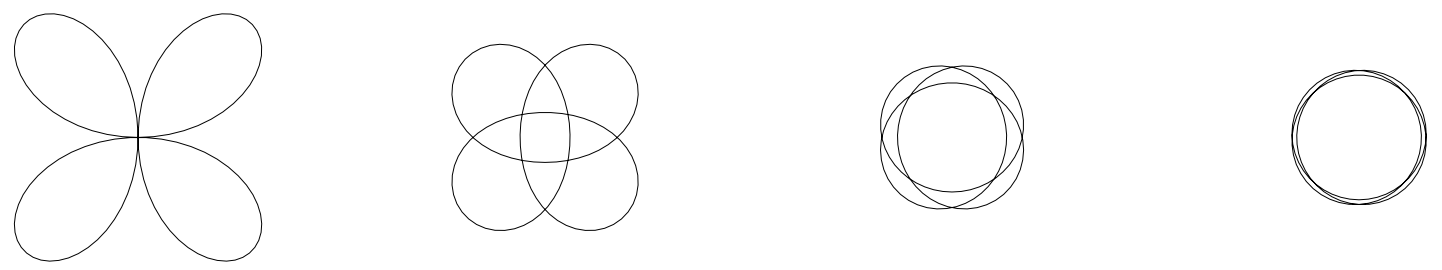

Figure 10: Surface diffusion flow for a rose. $\vec{X}(t)$ at times $t=0,0.02,0.05,0.075$.

ellipse with semiminor axis $R=0.75$, and let $\tau=0.5 h^{2}$ with $T=15$, by which time the numerical solutions have reached a circular "steady state". In Table 2 we report on the relative area loss compared to the area $a^{0}$ of the initial polygon, as well as the error $\left|a^{M}-a(0)\right|$ and the indicative error ||$\Gamma^{M}\left|-\lim _{t \rightarrow \infty}\right| \Gamma(t) \|$, i.e. the differences in area and in length to the true asymptotic solution $\vec{x}^{\star}:=\lim _{t \rightarrow \infty} \vec{x}(\cdot, t)$, which is given by a circle. We also report on the error $\left\|\vec{X}(T)-\vec{x}^{\star}\right\|_{L^{\infty}}:=\max _{j=1 \rightarrow N} \min _{\rho \in J}\left|\vec{X}^{M}\left(q_{j}\right)-\vec{x}^{\star}\right|$ between $\vec{X}(T)$ and the true asymptotic solution $\vec{x}^{\star}$. 


\begin{tabular}{r|c|c|c|c}
$N$ & $\left|a^{0}-a^{M}\right| /\left|a^{0}\right|$ & $\left|a^{M}-a(0)\right|$ & ||$\Gamma^{M}\left|-\lim _{t \rightarrow \infty}\right| \Gamma(t) \|$ & $\left\|\vec{X}(T)-\vec{x}^{\star}\right\|_{L^{\infty}}$ \\
\hline 16 & $4.4 \%$ & $9.2741 \mathrm{e}-01$ & $3.7046 \mathrm{e}-01$ & $4.7917 \mathrm{e}-02$ \\
32 & $1.3 \%$ & $2.6356 \mathrm{e}-01$ & $1.0482 \mathrm{e}-01$ & $1.4588 \mathrm{e}-02$ \\
64 & $0.4 \%$ & $7.3366 \mathrm{e}-02$ & $2.9446 \mathrm{e}-02$ & $5.1632 \mathrm{e}-03$ \\
128 & $0.1 \%$ & $2.0149 \mathrm{e}-02$ & $8.1808 \mathrm{e}-03$ & $2.4653 \mathrm{e}-03$ \\
256 & $0.03 \%$ & $5.5318 \mathrm{e}-03$ & $2.2717 \mathrm{e}-03$ & $1.7030 \mathrm{e}-03$ \\
512 & $0.008 \%$ & $1.5181 \mathrm{e}-03$ & $6.2799 \mathrm{e}-04$ & $1.4872 \mathrm{e}-03$
\end{tabular}

Table 2: Relative area loss and some errors with respect to the true asymptotic solution $\vec{x}^{\star}:=\lim _{t \rightarrow \infty} \vec{x}(\cdot, t)$.

\subsection{Willmore flow for curves}

As a first test, we repeated the computations for a true solution as given in Dziuk, Kuwert, and Schätzle (2002, p. 1241). An exact solution to (2.42), with $\lambda=0$, is given by

$$
\vec{x}(\rho, t)=(1+2 t)^{\frac{1}{4}}(\cos g(\rho), \sin g(\rho))^{T}, \quad \varkappa(\rho, t)=(1+2 t)^{-\frac{1}{4}},
$$

where $g(\rho)=2 \pi \rho+0.1 \sin (2 \pi \rho)$ in order to make the initial distribution of nodes nonuniform, and where we note that $\vec{x}_{t} \cdot \vec{\tau}=0$. Note furthermore that the true curvature vector is given by $\vec{\varkappa}(\rho, t)=-(1+2 t)^{-\frac{1}{4}}(\cos g(\rho), \sin g(\rho))^{T}$. The results can be seen in Table 3, where we report on the errors in $L^{\infty}\left(0, T ; L^{\infty}\left(J, \mathbb{R}^{(2)}\right)\right)$ for $T=1$ and $\tau=0.5 h^{2}$. We compare our results from $(2.45 \mathrm{a}, \mathrm{b})$, where, on noting that $\vec{N}_{0}^{T} \vec{N}_{0}$ is a diagonal matrix with strictly positive diagonal entries, we use as initial data

$$
\kappa^{0}:=-\left(\vec{N}_{0}^{T} \vec{N}_{0}\right)^{-1} \vec{N}_{0}^{T} \vec{A}_{0} \vec{X}^{0}
$$

to the corresponding version of the scheme in Dziuk, Kuwert, and Schätzle (2002), i.e. $(2.3 \mathrm{a}, \mathrm{b})$ with the last term on the left hand side of $(2.3 \mathrm{a})$ replaced by $-\lambda_{m}\left\langle\vec{\kappa}^{m+1}, \vec{\chi}\right\rangle_{m}^{h}$; with no redistribution of nodes. Note that we report on their scheme in two different columns, as with the original scheme $(2.3 \mathrm{a}, \mathrm{b})$ we could not quite reproduce the numbers reported in their paper. In particular, we report on the original scheme $(2.3 \mathrm{a}, \mathrm{b})$, "DKS (orig)", and a small modification, "DKS (mod)". The modified scheme uses $\left|\vec{\kappa}^{m+\frac{1}{2}}\right|^{2}$ instead than $\left|\vec{\kappa}^{m}\right|^{2}$ in $(2.3 \mathrm{a})$, where $\vec{\kappa}^{m+\frac{1}{2}} \in \underline{V}_{0}^{h}$ is such that

$$
\left\langle\vec{\kappa}^{m+\frac{1}{2}}, \vec{\eta}\right\rangle_{m}^{h}+\left\langle\nabla_{s} \vec{X}^{m}, \nabla_{s} \vec{\eta}\right\rangle_{m}=0 \quad \forall \vec{\eta} \in \underline{V}_{0}^{h} .
$$

We show the evolution of a lemniscate under the flow (2.42) with $\lambda=0$ and $\lambda=1$ in Figure 11. Note that no redistribution of nodes was necessary for $(2.45 \mathrm{a}, \mathrm{b})$, whereas the scheme $(2.3 \mathrm{a}, \mathrm{b})$ was not able to compute this evolution without a redistribution of nodes. The same experiment for the length preserving elastic flow, i.e. with the time dependent parameter $\lambda_{m}$ as in (2.46) is shown in Figure 12. The initial curve is a $2: 1$ lemniscate. The discretization parameters are $N=100$ and $\tau=10^{-3}$, the final time is $T=1$. The length of the original curve was almost exactly preserved (the length of the initial curve is 7.86 and that has grown by only $2.3 \times 10^{-3}$ at time $\left.t=T\right)$. The length preserving 


\begin{tabular}{|r|c|c|c|c|c|c|}
\hline & \multicolumn{3}{|c|}{ DKS (orig) } & \multicolumn{2}{c|}{ DKS (mod) } & \multicolumn{2}{c|}{$(2.45 \mathrm{a}, \mathrm{b})$} \\
$N$ & $\|\vec{X}-\vec{x}\|_{L^{\infty}}$ & $\|\vec{\kappa}-\vec{\varkappa}\|_{L^{\infty}}$ & $\|\vec{X}-\vec{x}\|_{L^{\infty}}$ & $\|\vec{\kappa}-\vec{\varkappa}\|_{L^{\infty}}$ & $\|\vec{X}-\vec{x}\|_{L^{\infty}}$ & $\|\kappa-\varkappa\|_{L^{\infty}}$ \\
\hline 10 & $2.7861 \mathrm{e}-01$ & $1.2994 \mathrm{e}-01$ & $9.8686 \mathrm{e}-03$ & $6.6960 \mathrm{e}-03$ & $2.2124 \mathrm{e}-01$ & $7.6410 \mathrm{e}-02$ \\
20 & $3.7365 \mathrm{e}-02$ & $1.7942 \mathrm{e}-02$ & $2.8856 \mathrm{e}-03$ & $3.2501 \mathrm{e}-03$ & $3.6298 \mathrm{e}-02$ & $8.2407 \mathrm{e}-03$ \\
40 & $8.3949 \mathrm{e}-03$ & $4.6565 \mathrm{e}-03$ & $7.9028 \mathrm{e}-04$ & $5.5311 \mathrm{e}-04$ & $8.5412 \mathrm{e}-03$ & $2.3799 \mathrm{e}-03$ \\
80 & $2.0410 \mathrm{e}-03$ & $1.0037 \mathrm{e}-03$ & $2.1042 \mathrm{e}-04$ & $2.1395 \mathrm{e}-04$ & $2.1143 \mathrm{e}-03$ & $4.4060 \mathrm{e}-04$ \\
160 & $5.0574 \mathrm{e}-04$ & $1.1518 \mathrm{e}-04$ & $5.4438 \mathrm{e}-05$ & $1.2143 \mathrm{e}-04$ & $5.2844 \mathrm{e}-04$ & $2.5524 \mathrm{e}-05$ \\
320 & $1.2602 \mathrm{e}-04$ & $4.1575 \mathrm{e}-05$ & $1.3859 \mathrm{e}-05$ & $2.4003 \mathrm{e}-05$ & $1.3224 \mathrm{e}-04$ & $6.6480 \mathrm{e}-06$ \\
640 & $3.1463 \mathrm{e}-05$ & $1.1178 \mathrm{e}-05$ & $3.4973 \mathrm{e}-06$ & $5.6171 \mathrm{e}-06$ & $3.3086 \mathrm{e}-05$ & $2.4659 \mathrm{e}-06$ \\
\hline
\end{tabular}

Table 3: Absolute errors $\|\vec{X}-\vec{x}\|_{L^{\infty}}$ and $\|\kappa-\varkappa\|_{L^{\infty}}$ for the test problem.
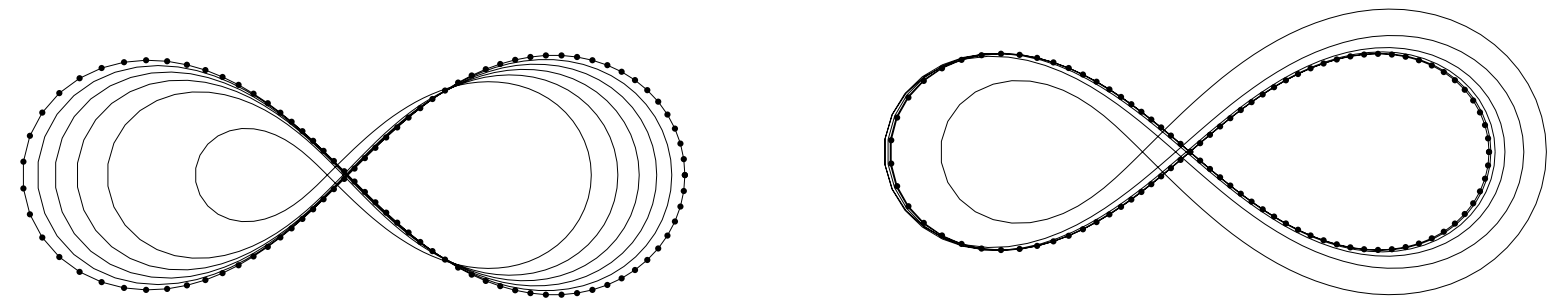

Figure 11: Willmore flow for a closed curve. On the left $\lambda=0$, on the right $\lambda=1$.

modification of (2.3a,b), see Dziuk, Kuwert, and Schätzle (2002, p. 1240), however, fails to compute this example without the redistribution of vertices. The solution at time $t=0.346$ can be seen on the right hand side of the figure.

A similar experiment for a $4: 1$ lemniscate and $T=4$ can be seen in Figure 13. Again, no redistribution was needed for scheme $(2.45 \mathrm{a}, \mathrm{b})$, while the scheme $(2.3 \mathrm{a}, \mathrm{b})$ without redistribution failed, this time at time $t=0.154$.

\subsection{Triple junctions}

In the first experiment for triple junctions, see Figure 14, we simulate how two initially elliptic bubbles approach the standard double bubble shape, where $\Gamma_{2}$ is a straight line and $\Gamma_{1}$ is the arc of a circle with $\Gamma_{3}$ its reflection, see Hutchings, Morgan, Ritoré, and Ros (2002). Throughout, we assume equal surface energies, $\sigma_{i}=1, i=1 \rightarrow K_{C}$, unless stated otherwise. A non equal area case can be seen on the right hand side of the same figure.

For the standard double bubble, we checked for our scheme $(2.26 \mathrm{a}, \mathrm{b})$, as $h \rightarrow 0$, the 

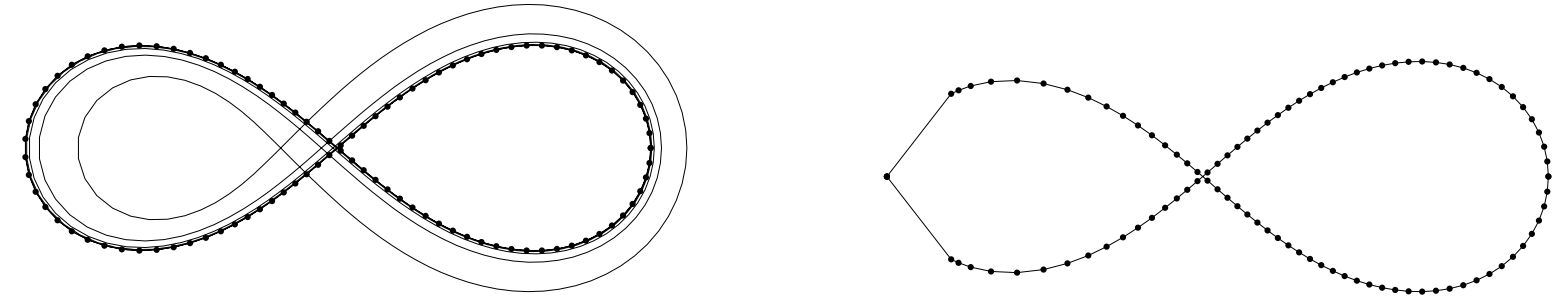

Figure 12: Length preserving elastic flow. Our scheme $(2.45 \mathrm{a}, \mathrm{b})$ on the left, the appropriately modified scheme $(2.3 \mathrm{a}, \mathrm{b})$ on the right.
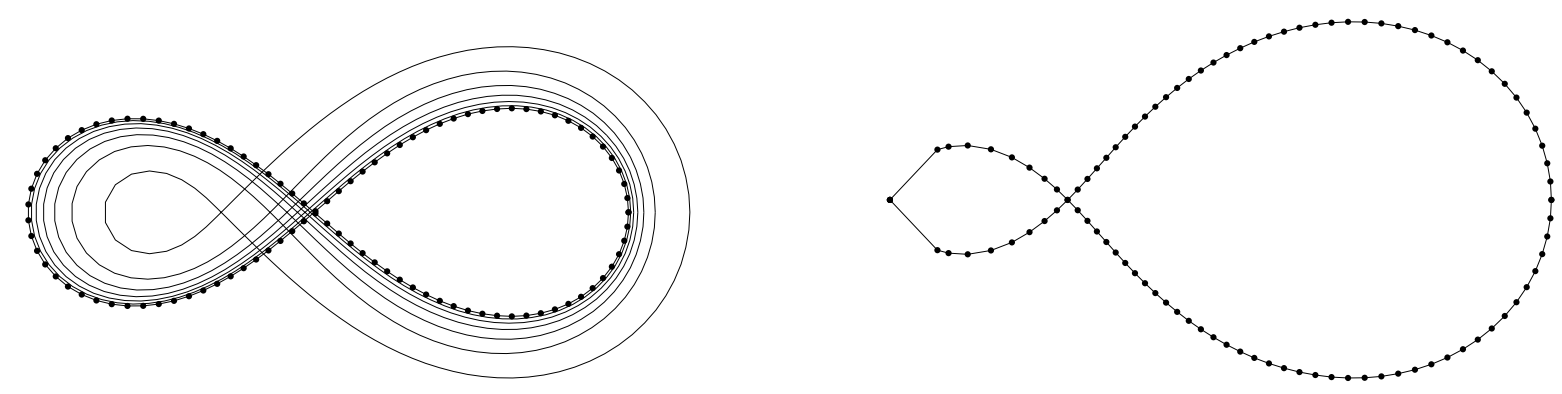

Figure 13: Length preserving elastic flow. Our scheme $(2.45 \mathrm{a}, \mathrm{b})$ on the left, the appropriately modified scheme $(2.3 \mathrm{a}, \mathrm{b})$ on the right.
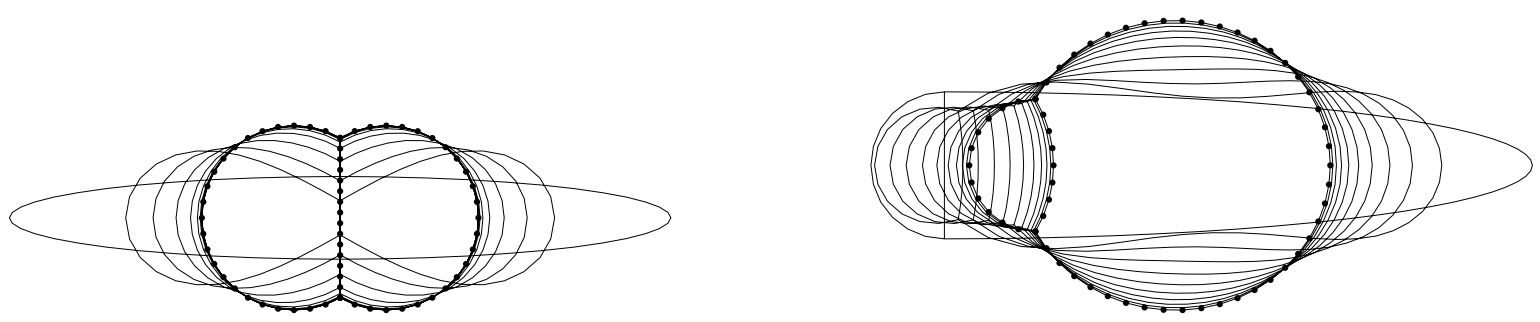

Figure 14: The standard double bubble, with a non equal area case on the right. 


\begin{tabular}{c|c|c|c}
$N$ & $h_{\vec{X}^{M}}$ & area loss & angles $\left(\theta_{1}, \theta_{2}, \theta_{3}\right)$ \\
\hline 32 & $2.6148 \mathrm{e}-02$ & $2.0280 \mathrm{e}-04$ & $(110.4,139.2,110.4)$ \\
64 & $1.3012 \mathrm{e}-02$ & $5.0898 \mathrm{e}-05$ & $(115.3,129.4,115.3)$ \\
128 & $6.1883 \mathrm{e}-03$ & $1.2261 \mathrm{e}-05$ & $(117.7,124.6,117.7)$ \\
256 & $3.0213 \mathrm{e}-03$ & $3.0659 \mathrm{e}-06$ & $(118.9,122.3,118.9)$ \\
512 & $1.4932 \mathrm{e}-03$ & $7.6653 \mathrm{e}-07$ & $(119.4,121.1,119.4)$ \\
1024 & $7.4231 \mathrm{e}-04$ & $1.9163 \mathrm{e}-07$ & $(119.7,120.6,119.7)$ \\
2048 & $3.7115 \mathrm{e}-04$ & $4.7909 \mathrm{e}-08$ & $(119.9,120.3,119.9)$ \\
4096 & $1.8531 \mathrm{e}-04$ & $1.1963 \mathrm{e}-08$ & $(119.9,120.1,119.9)$ \\
8192 & $9.2590 \mathrm{e}-05$ & $2.9908 \mathrm{e}-09$ & $(120.0,120.1,120.0)$
\end{tabular}

Table 4: Area loss and triple junction angles as $h \rightarrow 0$.

\begin{tabular}{c|c|c|c}
$N$ & $h_{\vec{X}^{M}}$ & relative area loss & angles $\left(\theta_{1}, \theta_{2}, \theta_{3}\right)$ \\
\hline 32 & $3.5583 \mathrm{e}-01$ & $0.3 \%$ & $(109.6,140.9,109.6)$ \\
64 & $1.7864 \mathrm{e}-01$ & $0.08 \%$ & $(114.9,130.2,114.9)$ \\
128 & $8.9415 \mathrm{e}-02$ & $0.02 \%$ & $(117.5,125.1,117.5)$ \\
256 & $4.4261 \mathrm{e}-02$ & $0.007 \%$ & $(118.8,122.5,118.8)$ \\
512 & $2.2132 \mathrm{e}-02$ & $0.002 \%$ & $(119.4,121.2,119.4)$
\end{tabular}

Table 5: Area loss and triple junction angles as $h \rightarrow 0$.

absolute area loss $\max _{i=1 \rightarrow 2}\left|a_{i}^{0}-a_{i}^{M}\right|$ and the observed angles $\theta:=\left(\theta_{1}, \theta_{2}, \theta_{3}\right)$ at the triple junctions, which, through symmetry, are the same at both triple junction points. Here $\theta$ is defined as the angles formed by the three curve segments meeting at a triple junction and $\theta_{i}, i=1 \rightarrow 3$, denotes the angle opposite the curve $\Gamma_{i}^{M}$ at a triple junction. The parameters were chosen as follows. The initial shape is a parameterization of the standard double bubble with two circles of radius 0.075 . We integrate until time $T=10^{-4}$ and used a uniform time step size $\tau=10^{-5} \frac{32}{N}$. For the results see Table 4 , where the maximal element length $h_{\vec{X}^{M}}$ at the final time is defined similarly to Table 2, and the area error is defined with respect to the true double bubble solution $\vec{x}$. Note that the relative area loss $\max _{i=1 \rightarrow 2} \frac{\left|a_{i}^{0}-a_{i}^{M}\right|}{\left|a_{i}^{0}\right|}$ for these experiments was always less than $10^{-5} \%$. In a more realistic experiment, we used as initial data two $3: 1$ ellipses with semiminor axis $R=0.75$, similarly to the left hand side of Figure 14, and integrated until time $T=2$. The results for a time step size of $\tau=0.5 h^{2}$ are displayed in Table 5 .

We note that for both sets of experiments one observes that the triple junction angles approach the true value $120^{\circ}$ as $h \rightarrow 0$, while the error in the area and the relative area loss tend to zero.

The considerations for the semidiscrete version of $(2.26 \mathrm{a}, \mathrm{b})$ in Remarks 2.4 and 2.5 are underlined by the following experiment. Using the parameters $N=64, \tau=10^{-3}$ and $T=1$ we investigate how an initial approximation of two semi-circles of radius $R=1$ 

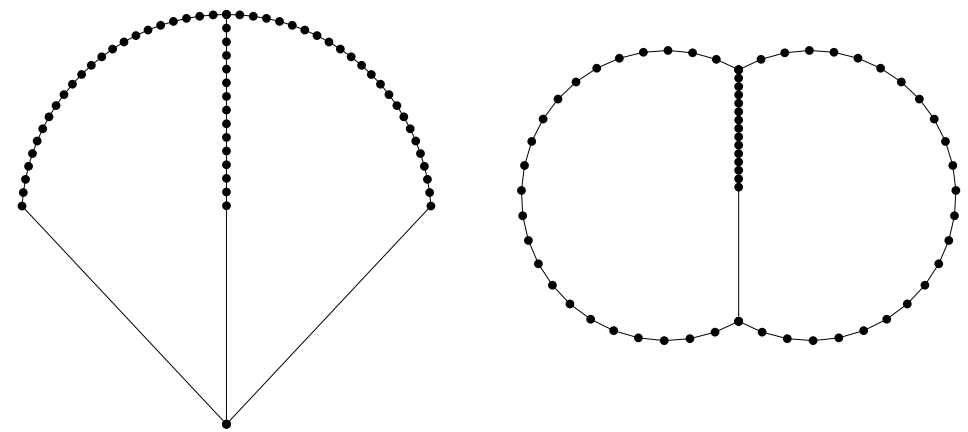

Figure 15: Tangential movement for an equal area double bubble. A plot of $\vec{X}(t)$ at times $t=0$ and $t=T$.
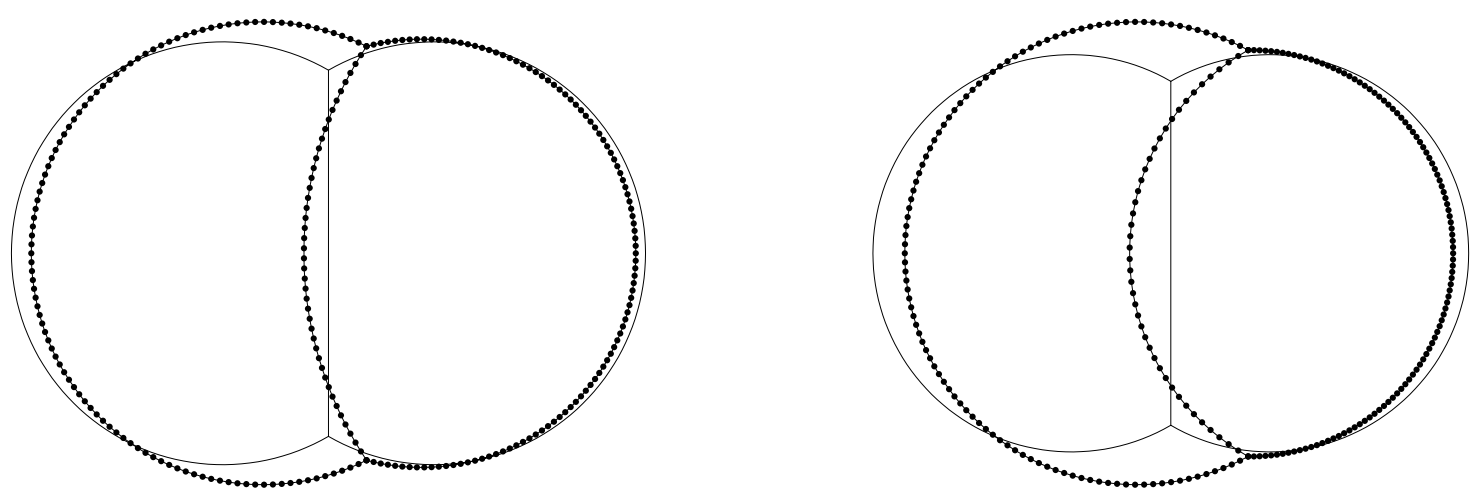

Figure 16: An equal area double bubble for surface energies $\left(1,1, \frac{3}{2}\right)$ and $\left(1,1, \frac{7}{4}\right)$.

with a straight line in between, where we uniformly parameterize only the upper half of each curve, is evolved by our scheme $(2.26 \mathrm{a}, \mathrm{b})$. As can be seen in Figure 15, the vertices on the two circular curve segments are equidistributed, while the nodes on the straight line segment experience no tangential movement. This is in line with the analysis for the semidiscrete scheme in Remarks 2.4 and 2.5, which predicts that on each curve $\Gamma_{i}$, $i=1 \rightarrow 3$, the vertices will be equidistributed as long as the elements on that curve are not locally parallel.

An equal area double bubble for different surface energies can be seen in Figure 16. The surface energies were chosen to be $\left(\sigma_{1}, \sigma_{2}, \sigma_{3}\right)=\left(1,1, \frac{3}{2}\right)$ and $\left(1,1, \frac{7}{4}\right)$, respectively. That means that the length of the curve $\Gamma_{3}$ is weighted more in the overall energy $|\Gamma|$, so that it will shorten during the evolution. For the parameters $N=256, T=1$ and $\tau=10^{-4}$, and starting from the standard double bubble with radii $R=1$, the observed angles at the triple junctions at time $T$ are $\theta=(136.8,140.7,82.5)$ and $\theta=(148.8,153,58.2)$ respectively. (Note that Young's law yields $\theta=(138.6,138.6,82.8)$ and $\theta=(151,151,57.9)$, respectively, for the exact solution.) An experiment for the surface energies $(1,1,2)$ can be seen in Figure 17. Here the observed angles at the triple junctions at time $T$ are $\theta=(173.4,178.9,7.8)$. However, one should note that the true steady state for this experiment would consist of only two circular curves, with the third one shrunk to a point. Of course, we cannot compute until that singularity. 

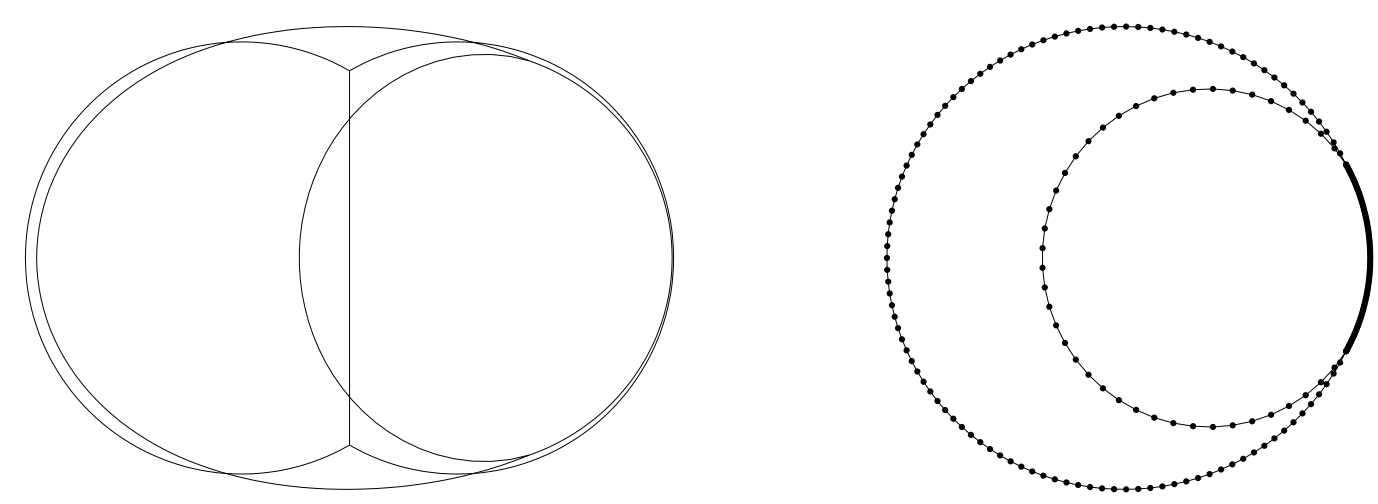

Figure 17: An equal area double bubble for surface energies $(1,1,2) . \quad \vec{X}(t)$ at times $t=0,0.1$ (left) and $t=T$ (right).
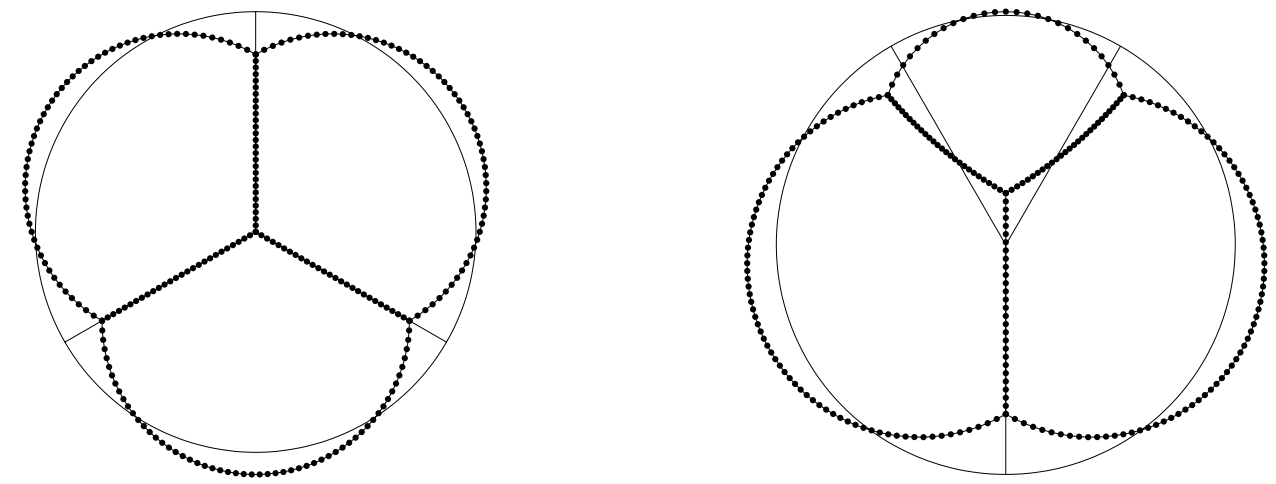

Figure 18: The standard triple bubble (left) and a non equal area triple bubble (right).

For the remaining experiments we always use equal surface energies. Examples with three and four enclosed areas, respectively, can be seen in Figures 18 and 19. Examples with five, six and seven enclosed areas can be seen in Figures 20, 21 and 22, respectively.

\section{Conclusions}

We have presented a fully practical finite element approximation for the motion by surface diffusion of curves in $\mathbb{R}^{2}$. Our scheme enjoys the property that no artificial redistribution of vertices is necessary in practice. To our knowledge, this is the first such scheme in the literature. Moreover, the presented scheme can easily be generalized to the case of multiple curves that meet at triple junction points. Also the case of mean curvature flow, $\mathcal{V}=\varkappa$, with and without triple junctions can be treated with the ideas developed in this paper. Moreover, nonlinear functions of mean curvature can equally be used as a driving 

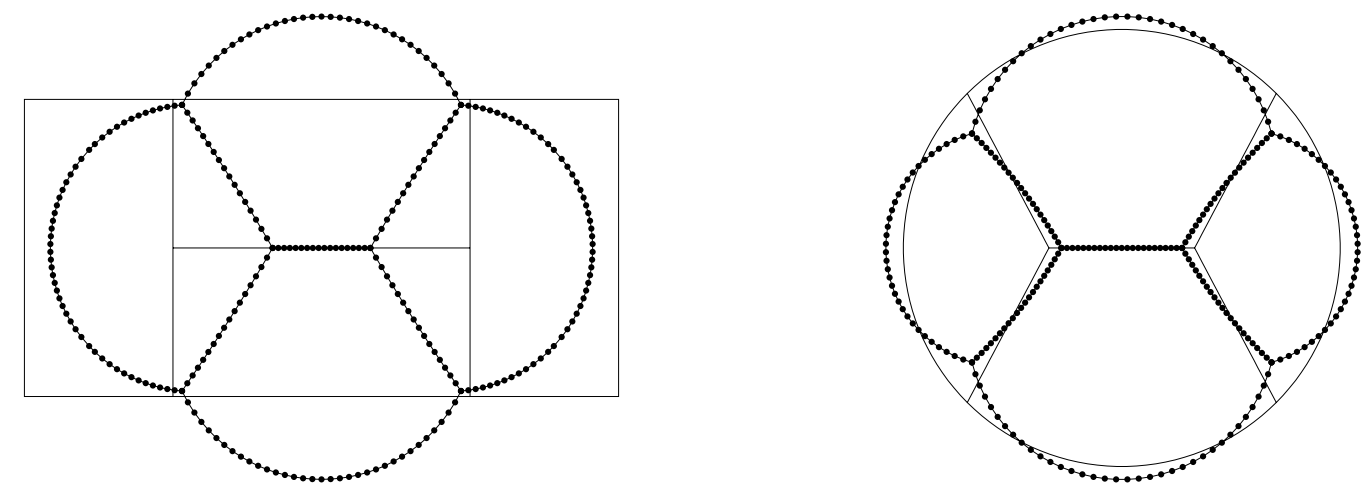

Figure 19: The standard quadruple bubble (left) and a non equal area quadruple bubble (right).
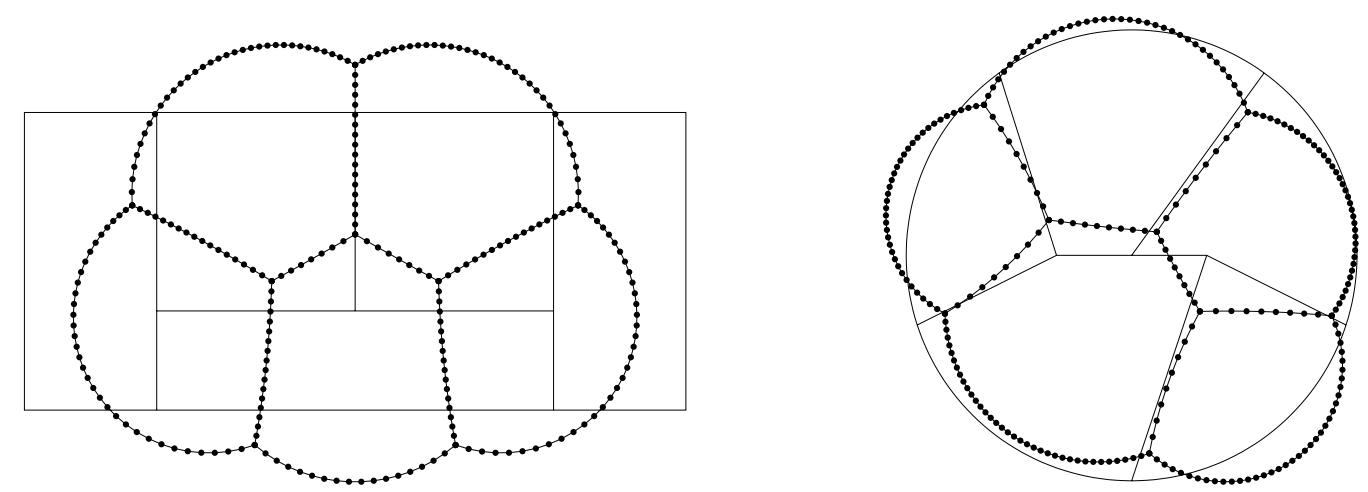

Figure 20: A standard quintic bubble (left) and a non equal area quintic bubble (right).
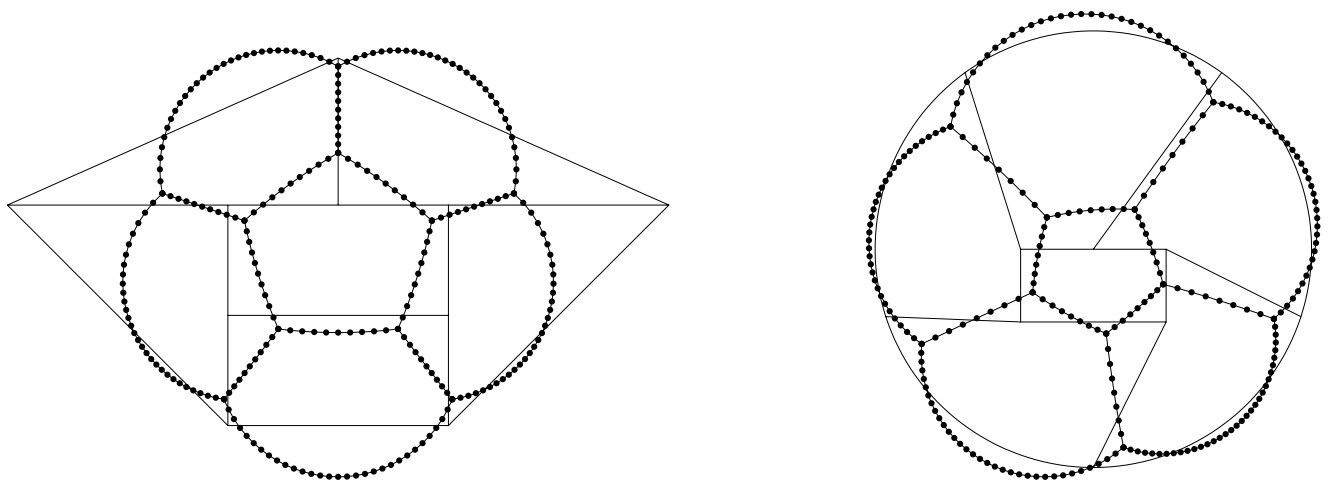

Figure 21: A standard sextic bubble (left) and a non equal area sextic bubble (right). 

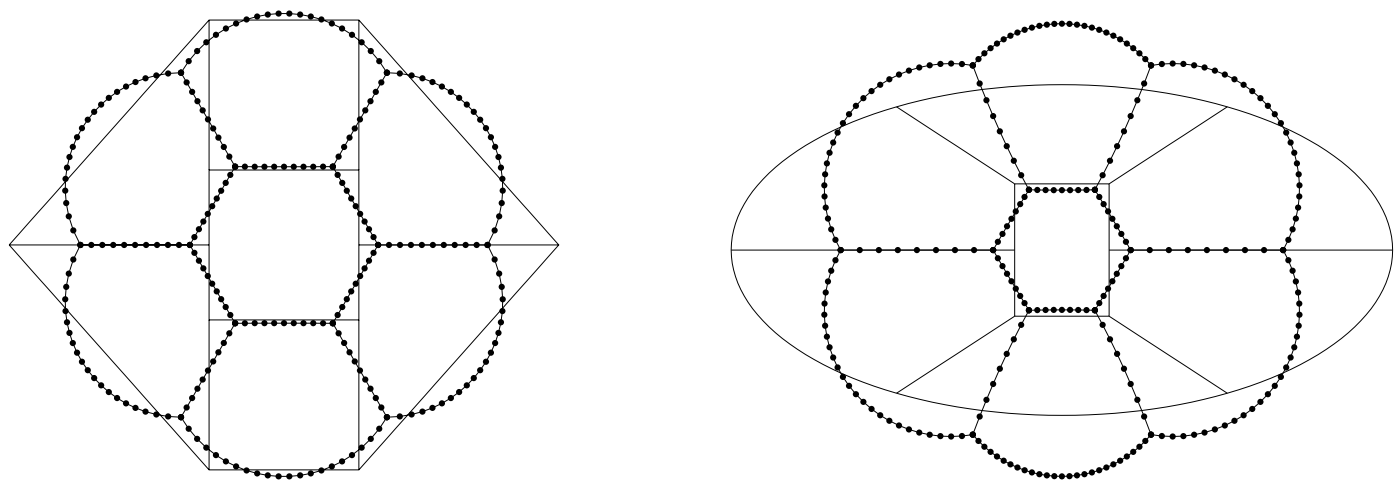

Figure 22: A standard septic bubble (left) and a non equal area septic bubble (right).

force. The equation $\mathcal{V}=f(\varkappa)$ can be discretized, for example, by

$$
\left\langle\frac{\vec{X}^{m+1}-\vec{X}^{m}}{\tau_{m}}, \chi \vec{\nu}^{m}\right\rangle_{m}^{h}-\left\langle f\left(\kappa^{m+1}\right), \chi\right\rangle_{m}^{h}=0 \quad \forall \chi \in W_{0}^{h},
$$

together with (2.2b), and so will also inherit the equidistribution property (2.16). Furthermore, the combined effect of motion by surface diffusion and motion by mean curvature can be modelled with a small change to our scheme. This and the additional effects of curves intersecting the external boundary of the domain will be studied in the forthcoming paper Barrett, Garcke, and Nürnberg (2006b).

Further topics of our future research related to the presented paper include the extension to fully anisotropic surface energies and to geometric flows of 2-dimensional hypersurfaces in $\mathbb{R}^{3}$, including mean curvature flow, surface diffusion and Willmore flow.

\section{Appendix}

Here we want to investigate the kernel of the operator $K A K$ in (2.35) for the case of an arbitrary number $K_{B}$ of enclosed areas. Note that we assume that at each triple junction exactly three curves meet and that we exclude any intersection of a curve with the external boundary. Hence we have that $6\left(K_{B}-1\right)=2 K_{C}=3 K_{T}$, where $K_{C}$ and $K_{T}$ are the number of curves and triple junction points, respectively.

The definitions (2.34) are trivially extended to the general case, while (2.33) needs to be replaced by

$$
K: \mathbb{R}^{N} \rightarrow \mathbb{X}:=\left\{\left(z_{1}, \ldots, z_{K_{C}}\right) \in \mathbb{R}^{N}: \sum_{i=1}^{3}(-1)^{p_{j, i_{j}}} \sigma_{i_{j}}\left[z_{i_{j}}\right]_{p_{j, i_{j}} N_{i_{j}}}=0 \quad j=1 \rightarrow K_{T}\right\},
$$

where now $N:=\sum_{i=1}^{K_{C}}\left(N_{i}+1\right)$ and $p_{j, i_{j}} \in\{0,1\}, i=1 \rightarrow 3, j=1 \rightarrow K_{T}$, are defined as in Remark 2.6. 
We now want to find a basis for the space $E \subset \mathbb{X} \subset \mathbb{R}^{N}$, where ker $K A K=\operatorname{ker} K \oplus E \equiv$ $\mathbb{X}^{\perp} \oplus E$. For simplicity we assume that $\sigma_{i}=1, i=1 \rightarrow K_{C}$. Then it is clear that the $K_{C}$ vectors $v_{1}=\left(1^{1}, 0, \ldots, 0\right), v_{2}=\left(0,1^{2}, 0, \ldots, 0\right), \ldots, v_{K_{C}}=\left(0, \ldots, 0,1^{K_{C}}\right) \in \mathbb{R}^{N}$ form a basis of $\operatorname{ker} A$. Furthermore $E=\operatorname{ker} A \cap \mathbb{X}$.

Lemma. A.1. It holds that $\operatorname{dim} E=K_{B}$. A possible basis of $E$ is given by $e_{i}:=$ $\left(\alpha_{i}^{1} 1^{1}, \ldots, \alpha_{i}^{K_{C}} 1^{K_{C}}\right), i=1 \rightarrow K_{B}$, where $\alpha_{i}^{j} \in\{-1,0,1\}$ are such that for $\alpha_{i}^{j} \neq 0$, $\vec{X}_{j}^{m}\left(\frac{1+\alpha_{i}^{j}}{2} \rho+\frac{1-\alpha_{i}^{j}}{2}(1-\rho)\right)$ parameterizes a segment of the boundary of bubble $i$ clock-wise, such that the union of all these segments yields the whole boundary of bubble $i$.

Proof. On noting (A.1) it is clear that by construction $e_{i} \in \mathbb{X}$ and $e_{i} \in \operatorname{ker} A$, for all $i=1 \rightarrow K_{B}$. It is also easy to see that the $\left\{e_{i}\right\}_{i=1}^{K_{B}}$ are linearly independent. It remains to show that $\operatorname{dim} E=K_{B}$.

Let $\mathcal{C} \in \mathbb{R}^{K_{T} \times K_{C}}$ be the matrix such that

$$
e:=\left(\alpha^{1} 1^{1}, \ldots, \alpha^{K_{C}} 1^{K_{C}}\right) \in E \equiv \operatorname{ker} A \cap \mathbb{X} \quad \Longleftrightarrow \quad \mathcal{C} \underline{\alpha}=\underline{0},
$$

where $\underline{\alpha}:=\left(\alpha^{1}, \ldots, \alpha^{K_{C}}\right) \in \mathbb{R}^{K_{C}}$. That is, $\mathcal{C}$ describes the constraints in (A.1) and each row has exactly three non-zero entries (of modulus one), and each column has exactly two nonzero entries that add up to zero.

The latter property immediately yields that $\operatorname{rank} \mathcal{C} \leq K_{T}-1$. We will now show that $\operatorname{rank} \mathcal{C} \geq K_{T}-1$. Let $\mathcal{D} \in \mathbb{R}^{\left(K_{T}-1\right) \times K_{C}}$ denote the first $K_{T}-1$ rows of $\mathcal{C}$ and assume

$$
\mathcal{D}^{T} \underline{\beta}=\underline{0}
$$

for $\beta \in \mathbb{R}^{K_{T}-1}$. For a fixed $i \in\left\{1, \ldots, K_{T}-1\right\}$, let $\left(\ell_{1}, \ldots, \ell_{L}\right) \in\left\{1, \ldots, K_{T}\right\}^{L}$, with $\ell_{1}=K_{T}$ and $\ell_{L}=i$ denote a path within the given network of curves connecting triple junction point $\Lambda_{K_{T}}$ to point $\Lambda_{i}$. As we consider a connected network of curves, such a path always exists. It is now straightforward to show by induction that it follows from (A.3) that $\beta_{\ell_{j}}=0$ for all $j=1 \rightarrow L$, and in particular $\beta_{\ell_{L}}=\beta_{i}=0$.

Repeating this argument for all $i=1 \rightarrow K_{T}-1$ shows that (A.3) implies $\beta=\underline{0}$. Hence $K_{T}-1=\operatorname{rank} \mathcal{D} \geq \operatorname{rank} \mathcal{C}$. Thus we have that $\operatorname{rank} \mathcal{C}=K_{T}-1$ and, on recalling (A.2), it holds that $\operatorname{dim} E=K_{C}-\operatorname{rank} \mathcal{C}=K_{C}-\left(K_{T}-1\right)=3\left(K_{B}-1\right)-2\left(K_{B}-1\right)+1=K_{B}$.

\section{References}

Averbuch, A., M. Israeli, and I. Ravve (2003). Electromigration of intergranular voids in metal films for microelectronic interconnects. J. Comput. Phys. 186, 481-502.

Bänsch, E., P. Morin, and R. H. Nochetto (2004). Surface diffusion of graphs: variational formulation, error analysis, and simulation. SIAM J. Numer. Anal. 42, 77379 . 
Bänsch, E., P. Morin, and R. H. Nochetto (2005). A finite element method for surface diffusion: the parametric case. J. Comput. Phys. 203(1), 321-343.

Barrett, J. W., H. Garcke, and R. Nürnberg (2006a). Finite element approximation of a phase field model for multicomponent surface diffusion. (in preparation).

Barrett, J. W., H. Garcke, and R. Nürnberg (2006b). On the variational approximation of combined second and fourth order geometric evolution equations. Preprint No. 07/2006, University Regensburg.

Barrett, J. W., R. Nürnberg, and V. Styles (2004). Finite element approximation of a phase field model for void electromigration. SIAM J. Numer. Anal. 42, 738-772.

Bower, A. F. and D. Craft (1998). Analysis of failure mechanisms in the interconnect lines of microelectronic circuits. Fat. Frac. Eng. Mat. Struct. 21, 611-630.

Bronsard, L. and B. T. R. Wetton (1995). A numerical method for tracking curve networks moving with curvature motion. J. Comput. Phys. 120(1), 66-87.

Chopp, D. L. and J. A. Sethian (1999). Motion by intrinsic Laplacian of curvature. Interfaces Free Bound. 1, 107-123.

Davi, F. and M. E. Gurtin (1990). On the motion of a phase interface by surface diffusion. Z. Angew. Math. Phys. 41, 782-811.

Deckelnick, K., G. Dziuk, and C. M. Elliott (2003). Error analysis of a semidiscrete numerical scheme for diffusion in axially symmetric surfaces. SIAM J. Numer. Anal. 41(6), 2161-2179.

Deckelnick, K., G. Dziuk, and C. M. Elliott (2005a). Computation of geometric partial differential equations and mean curvature flow. Acta Numer. 14, 139-232.

Deckelnick, K., G. Dziuk, and C. M. Elliott (2005b). Fully discrete finite element approximation for anisotropic surface diffusion of graphs. SIAM J. Numer. Anal. 43(3), 1112-1138.

Di Carlo, A., M. E. Gurtin, and P. Podio-Guidugli (1992). A regularized equation for anisotropic motion-by-curvature. SIAM J. Appl. Math. 52(4), 1111-1119.

Droske, M. and M. Rumpf (2004). A level set formulation for Willmore flow. Interfaces Free Bound. 6(3), 361-378.

Dziuk, G. (1991). An algorithm for evolutionary surfaces. Numer. Math. 58(6), 603611.

Dziuk, G. (1999). Numerical schemes for the mean curvature flow of graphs. In Variations of domain and free-boundary problems in solid mechanics (Paris, 1997), Volume 66 of Solid Mech. Appl., pp. 63-70. Dordrecht: Kluwer Acad. Publ.

Dziuk, G., E. Kuwert, and R. Schätzle (2002). Evolution of elastic curves in $\mathbb{R}^{n}$ : existence and computation. SIAM J. Math. Anal. 33, 1228-1245.

Elliott, C. M. and H. Garcke (1997). Existence results for diffusive surface motion laws. Adv. Math. Sci. Appl. 7, 465-488.

Escher, J., H. Garcke, and K. Ito (2003). Exponential stability for a mirror-symmetric three phase boundary motion by surface diffusion. Math. Nachr. 257, 3-15. 
Escher, J., U. F. Mayer, and G. Simonett (1998). The surface diffusion flow for immersed hypersurfaces. SIAM J. Math. Anal. 29, 1419-1433.

Fried, E. and M. E. Gurtin (2003). The role of the configurational force balance in the nonequilibrium epitaxy of films. Mechanics and Physics of Solids 51, 487-517.

Garcke, H. and A. Novick-Cohen (2000). A singular limit for a system of degenerate Cahn-Hilliard equations. Adv. Differential Equations 5, 401-434.

Gurtin, M. E. and M. E. Jabbour (2002). Interface evolution in three dimensions with curvature-dependent energy and surface diffusion: interface-controlled evolution, phase transitions, epitaxial growth of elastic films. Arch. Ration. Mech. Anal. 163(3), 171-208.

Hestenes, M. R. (1975). Pseudoinverses and conjugate gradients. Comm. ACM 18, 40-43. Collection of articles honoring Alston S. Householder.

Hutchings, M., F. Morgan, M. Ritoré, and A. Ros (2002). Proof of the double bubble conjecture. Ann. of Math. (2) 155(2), 459-489.

Li, Z., H. Zhao, and H. Gao (1999). A numerical study of electro-migration voiding by evolving level set functions on a fixed cartesian grid. J. Comput. Phys. 152, 281-304.

Merriman, B., J. K. Bence, and S. J. Osher (1994). Motion of multiple functions: a level set approach. J. Comput. Phys. 112(2), 334-363.

Mikula, K. and D. Ševčovič (2001). Evolution of plane curves driven by a nonlinear function of curvature and anisotropy. SIAM J. Appl. Math. 61(5), 1473-1501.

Mikula, K. and D. Ševčovič (2005). Tangentially stabilized Lagrangian algorithm for elastic curve evolution driven by intrinsic Laplacian of curvature. In A. Handlovicova, Z. Kriva, K. Mikula, and D. Ševčovič (Eds.), ALGORITMY 2005, pp. 32-41. Slovak University of Technology.

Mullins, W. W. (1957). Theory of thermal grooving. J. Appl. Phys. 28, 333-339.

Neubauer, R. (2002). Ein Finiteelementeansatz für Krümmungsfluß von unter Tripelpunktbedingungen verbundenen Kurven. Master's thesis, University Bonn, Bonn.

Smith, K. A., F. J. Solis, and D. L. Chopp (2002). A projection method for motion of triple junctions by levels sets. Interfaces Free Bound. 4 (3), 263-276.

Thaddey, B. (1999). Numerik für die Evolution von Kurven mit Tripelpunkt. Master's thesis, University Freiburg, Freiburg.

Zhao, H.-K., B. Merriman, S. Osher, and L. Wang (1998). Capturing the behavior of bubbles and drops using the variational level set approach. J. Comput. Phys. 143(2), $495-518$. 